\title{
Conserved chromatin and repetitive patterns reveal slow genome evolution in frogs
}

\author{
Jessen V. Bredeson ${ }^{1 \dagger}$, Austin B. Mudd ${ }^{1 \dagger}$, Sofia Medina-Ruiz ${ }^{1 \dagger}$, Therese Mitros ${ }^{1}$, Owen K.
} Smith $^{2}$, Kelly E. Miller ${ }^{1}$, Jessica B. Lyons ${ }^{1,3}$, Sanjit S. Batra ${ }^{4}$, Joseph Park ${ }^{1}$, Kodiak C. Berkoff ${ }^{1}$, Christopher Plott $^{5}$, Jane Grimwood ${ }^{5}$, Jeremy Schmutz ${ }^{5}$, Guadalupe Aguirre-Figueroa ${ }^{2}$, Mustafa K. Khokha ${ }^{6}$, Maura Lane ${ }^{6}$, Isabelle Philipp ${ }^{1}$, Mara Laslo ${ }^{7}$, James Hanken ${ }^{7}$, Gwenneg Kerdivel ${ }^{8}$, Nicolas Buisine ${ }^{8}$, Laurent M. Sachs ${ }^{8}$, Daniel R. Buchholz ${ }^{9}$, Taejoon Kwon ${ }^{10}$, Heidi SmithParker $^{11}$, Marcos Gridi-Papp ${ }^{12}$, Michael J. Ryan ${ }^{11}$, Robert. D. Denton ${ }^{13}$, John H. Malone ${ }^{13}$, John B. Wallingford ${ }^{14}$, Aaron F. Straight ${ }^{2}$, Rebecca Heald ${ }^{1}$, Dirk Hockemeyer ${ }^{1,3,16}$, Richard M. Harland ${ }^{1}$, Daniel S. Rokhsar ${ }^{1,3,15,16,{ }^{*}}$

${ }^{1}$ Department of Molecular and Cell Biology, Weill Hall, University of California, Berkeley, CA, 94720, USA. ${ }^{2}$ Department of Biochemistry, Stanford University School of Medicine, 279 Campus Drive, Beckman Center 409, Stanford, CA, 94305-5307, USA.

${ }^{3}$ Innovative Genomics Institute, University of California, Berkeley, CA, 94720, USA.

${ }^{4}$ Computer Science Division, University of California Berkeley, 2626 Hearst Avenue, Berkeley, CA, 94720, USA.

${ }^{5}$ Genome Sequencing Center, HudsonAlpha Institute for Biotechnology, Huntsville, AL, USA.

${ }^{6}$ Pediatric Genomics Discovery Program, Departments of Pediatrics and Genetics, Yale University School of Medicine, 333 Cedar Street, New Haven, CT, 06510, USA.

${ }^{7}$ Department of Organismic and Evolutionary Biology, and Museum of Comparative Zoology, Harvard University, Cambridge, MA, 02138, USA.

${ }^{8}$ Département Adaptation du Vivant, UMR 7221 CNRS, Muséum National d'histoire Naturelle, Paris, France.

${ }^{9}$ Department of Biological Sciences, University of Cincinnati, Cincinnati, OH, USA.

${ }^{10}$ Department of Biomedical Engineering, Ulsan National Institute of Science and Technology, Ulsan, 44919, Republic of Korea.

${ }^{11}$ Department of Integrative Biology, Patterson Labs, 2401 Speedway, University of Texas, Austin, TX, 78712, USA.

${ }^{12}$ Department of Biological Sciences, University of the Pacific, 3601 Pacific Avenue, Stockton, CA, 95211, USA.

${ }^{13}$ Department of Molecular and Cell Biology and Institute of Systems Genomics, University of Connecticut, 181 Auditorium Road, Unit 3197, Storrs, CT, 06269, USA.

${ }^{14}$ Department of Molecular Biosciences, Patterson Labs, 2401 Speedway, The University of Texas at Austin, Austin, TX, 78712, USA.

${ }^{15}$ Okinawa Institute of Science and Technology Graduate University, Onna, Okinawa, 9040495, Japan.

${ }^{16}$ Chan-Zuckerberg BioHub, 499 Illinois Street, San Francisco, CA, 94158, USA.

${ }^{\dagger}$ These authors contributed equally

${ }^{*}$ Corresponding authors 


\section{Abstract}

2 Frogs are an ecologically diverse and phylogenetically ancient group of living amphibians that include important vertebrate cell and developmental model systems, notably the genus Xenopus. Here we report a high-quality reference genome sequence for the western clawed frog, Xenopus tropicalis, along with draft chromosome-scale sequences of three distantly related emerging model frog species, Eleutherodactylus coqui, Engystomops pustulosus and Hymenochirus boettgeri. Frog chromosomes have remained remarkably stable since the Mesozoic Era, with limited Robertsonian (i.e., centric) translocations and end-to-end fusions found among the smaller chromosomes. Conservation of synteny includes conservation of centromere locations, marked by centromeric tandem repeats associated with Cenp-a binding, surrounded by pericentromeric LINE/L1 elements. We explored chromosome structure across frogs, using a dense meiotic linkage map for $X$. tropicalis and chromatin conformation capture (HiC) data for all species. Abundant satellite repeats occupy the unusually long ( 20 megabase) terminal regions of each chromosome that coincide with high rates of recombination. Both embryonic and differentiated cells show reproducible association of

17 centromeric chromatin, and of telomeres, reflecting a Rabl configuration similar to the

18 "bouquet" structure of meiotic cells. Our comparative analyses reveal 13 conserved ancestral anuran chromosomes from which contemporary frog genomes were constructed. 


\section{Main Text}

\section{Introduction}

23 Amphibians are widely used models in developmental and cell biology ${ }^{1-5}$, and their importance

24 extends to the fields of infectious disease, ecology, pharmacology, environmental health, and biological diversity ${ }^{6-10}$. While the principal model systems belong to the genus Xenopus (notably the diploid western clawed frog $X$. tropicalis and the paleo-allotetraploid African clawed frog $X$.

27 laevis, other amphibian models have increasingly been introduced due to their diverse developmental, cell biological, physiological, and behavioral adaptations ${ }^{11-14}$.

While genome evolution has been extensively studied in mammals ${ }^{15}$ and birds $^{16,17}$, the relative

31 lack of phylogenetically diverse chromosome-scale frog genomes has limited the study of

32 genome evolution in anuran amphibians. Here, we report a high-quality assembly for $X$.

33 tropicalis and three new chromosome-scale genome assemblies for the direct-developing

34 Puerto Rican coquí (Eleutherodactylus coqui), the túngara frog (Engystomops pustulosus),

35 which is a model for vocalization, and the Zaire dwarf clawed frog (Hymenochirus boettgeri),

36 which has an unusually small embryo and is a model for regulation of cell and body sizes.

37 Genome assemblies are essential resources for further work to exploit the experimental

38 possibilities of these diverse animals. The new high quality $X$. tropicalis genome upgrades

39 previous draft assemblies ${ }^{18,19}$ and our new genomes complement draft chromosome-scale

40 sequences for the African clawed frog ${ }^{20}$ (Xenopus laevis), the African bullfrog ${ }^{21}$ (Pyxicephalus

41 adspersus), the Leishan moustache toad ${ }^{22}$ (Leptobrachium leishanense), the Ailao moustache

$42 \operatorname{toad}^{23}$ (Leptobrachium [Vibrissaphora] ailaonicum), and Asiatic toad ${ }^{24}$ (Bufo gargarizans), as

43 well as scaffold- and contig-scale assemblies for other species ${ }^{25}$. The rapidly increasing number 
44 of chromosome-scale genome assemblies makes anurans ripe for comparative genomic and

45 evolutionary analysis.

46

47 Chromosome number variation among frogs is limited ${ }^{26}$. Based on cytological ${ }^{27,28}$ and sequence 48 comparisons ${ }^{18,20,26,29,30}$ most frogs have $n \sim 10-12$ pairs of chromosomes. The constancy of the

49 frog karyotype is in contrast with the more dramatic variation seen across mammals ${ }^{15,31}$, which

50 as a group is considerably younger than frogs. The constancy of the frog karyotype parallels the

51 static karyotypes of birds ${ }^{16}$, although birds typically have nearly three times more chromosomes

52 than frogs, including numerous microchromosomes (among frogs, only the basal Ascaphus ${ }^{32}$

53 has microchromosomes). Despite the stable frog chromosome number, however, fusions,

54 fissions, and other inter-chromosomal rearrangements do occur, and we can use comparisons

55 among chromosome-scale genome sequences to (1) infer the ancestral chromosomal elements,

56 (2) determine the rearrangements that have occurred along frog phylogeny, and (3) characterize

57 the patterns of chromosomal change among frogs. These findings of conserved synteny among

58 frogs are consistent with prior demonstrations of conservation between Xenopus tropicalis with

59 other tetrapods, including human and chicken ${ }^{18,33}$.

61 Since frog karyotypes are so highly conserved, $X$. tropicalis can be used as a model for studying

62 chromosome structure, chromatin interaction, and recombination for the entire clade. Features

63 that can be illuminated at the sequence level include the structure and organization of

64 centromeres and nature of the unusually long subtelomeres relative to mammals (frog

65 subtelomeres are $\sim 20$ megabases, compared with the mammalian subtelomeres that are

66 typically shorter than a megabase). The extended subtelomeres of frogs form interacting

67 chromatin structures in interphase nuclei that reflect three-dimensional intra-chromosome and

68 inter-chromosome subtelomeric contacts, which are consistent with a Rabl configuration. As in

69 other animals, subtelomeres of frogs have an elevated GC content and recombination rate. 
70 Here we show that the unusually high enrichment of recombination in the subtelomeres likely

71 reflects similar structural and functional properties in other vertebrates, though the quality of the

72 assembly reveals that the length of subtelomeres, enrichment of transposon subsequences by

73 unequal crossing over, and high recombination rates are considerably greater than in mammals.

74 We use Cenp-a binding at satellites to confirm centromere identity and extend the predictive

75 power of the repeat structures to centromeres of other frogs. We address the unusually high

76 recombination rate in subtelomeric regions, correlating with the landscape of base composition

77 and transposons. Over the 200 million years of evolution that we address here, centromeres

78 have generally been stable, but the few karyotypic changes reveal the predominant

79 Robertsonian translocations at centromeric regions; we also document the slow degeneration

80 that occurs to inactivated centromeres and fused telomeres, changes that are obscured in

81 animals with rapidly evolving karyotypes.

\section{New frog genome sequences}

85 High-quality chromosome-scale genome assembly for $X$. tropicalis

86 To establish a high-quality chromosomal reference genome sequence to study the structure and

87 organization of Xenopus tropicalis chromosomes and for comparisons with other frog genomes,

88 we integrated multiple sequencing technologies, including Single-Molecule Real-Time long

89 reads (SMRT sequencing; Pacific Biosciences), linked read sets (10x Genomics), short-read

90 shotgun sequencing, in vivo chromatin conformation capture, and meiotic mapping, combined

91 with previously generated dideoxy shotgun sequence (Supplementary Data 1, Supplementary

92 Figs. 1A-D and 2, and Supplementary Notes 1 and 2). New sequences were generated from

93 17th generation individuals from the same inbred Nigerian line that was used in the original 
94 Sanger shotgun sequencing ${ }^{33}$. The completeness, protein coding capacity, repeat structure and

95 sequence variation are discussed in supplementary information (Supplementary Figs. 1-6 and

Supplementary Tables 1-4) providing the basis for comparisons.

98 The new $\mathrm{v} 10$ reference assembly spans $1,448.4 \mathrm{Mb}$ and is substantially more complete than the 99 previous v9 sequence ${ }^{18}$, assigning $219.2 \mathrm{Mb}$ more sequence to chromosomes (Supplementary

100 Table 1). The v10 assembly is also far more contiguous, with half of the sequence contained in

10132 contigs longer than $14.6 \mathrm{Mb}$ (vs. $71.0 \mathrm{~kb}$ in v9). The assembly captures $99.6 \%$ of known

102 coding sequence (Supplementary Table 2, Supplementary Note 2). We found that the

103 fragmented quality of earlier assemblies was due in part to the fact that $68.3 \mathrm{Mb}(4.71 \%)$ of the

104 genome was not sampled by the $8 \times$ redundant Sanger dideoxy whole-genome shotgun

105 dataset $^{33}$ (Supplementary Fig. 3, Supplementary Note 2). These missing sequences,

106 apparently due to non-uniformities in shotgun cloning and/or sequences (Supplementary Fig.

107 1E), are distributed across an estimated 140.5k blocks of mean size 485.7 bp (longest $50.0 \mathrm{~kb}$ )

108 on the new reference assembly and capture an additional 6,774 protein-coding exons

109 (Supplementary Fig. 1F-G). The enhanced contiguity of v10 is accounted for by the relatively

110 uniform coverage of PacBio long-read sequences on the genome, as expected from other

111 studies ${ }^{34-37}$. Most remaining gaps are in highly repetitive and satellite-rich centromeres and

112 subtelomeric regions (see below) (Supplementary Figs. 1H and 3).

\section{Additional chromosome-scale frog genomes}

115 To assess the evolution of chromosome structure across a diverse set of frogs, we generated

116 chromosome-scale genome assemblies for three new emerging model species, including the

117 Zaire dwarf clawed frog Hymenochirus boettgeri (a member of the family Pipidae along with

118 Xenopus spp.) and two neobratrachians: the Puerto Rican coquí Eleutherodactylus coqui (family 
Eleutherodactylidae) and the túngara frog Engystomops pustulosus (family Leptodactylidae).

These chromosome-scale draft genomes were primarily assembled from short-read datasets

121 and chromatin conformation capture $(\mathrm{HiC})$ data (Supplementary Data 1, Supplementary

122 Table 5, Supplementary Note 3). To further expand the scope of our comparisons, we also

123 updated the assemblies of two recently published frog genomes: the African bullfrog

124 Pyxicephalus adspersus ${ }^{21}$ from the neobatrachian family Pyxicephalidae, and the Ailao

125 moustache toad Leptobrachium (Vibrissaphora) ailaonicum ${ }^{22}$ from the family Megophryidae

126 (Supplementary Fig. 7, Supplementary Note 3). These species span the pipanuran clade,

127 which comprises all extant frogs except for a small number of phylogenetically basal taxa, such

128 as Ascaphus ${ }^{38}$.

The chromosome numbers of the new assemblies agree with previously described karyotypes for $E$. coqui $^{39}(2 n=26)$ and E. pustulosus ${ }^{40}(2 n=22)$. The literature for $H$. boettgeri, however, is more equivocal, with reports ${ }^{41,42}$ of $2 n=20-24$. The $n=9$ chromosomes in our $H$. boettgeri assembly are consistent with our chromosome spreads (Supplementary Fig. 7A). The karyotype variability in the published literature and discrepancy with karyotypes of our $H$. boettgeri samples may be the result of cryptic sub-populations within this species, or segregating chromosome polymorphisms.

139 The improved $X$. tropicalis genome encodes an estimated 25,016 protein-coding genes

140 (Supplementary Table 3), which we predicted by taking advantage of 8,580 full-length-insert $X$.

141 tropicalis cDNAs from the "Mammalian" Gene Collection"33 (MGC), 1.27 million Sanger-

142 sequenced expressed sequence tags ${ }^{33}$ (ESTs), and $334.5 \mathrm{Gbp}$ of RNA-seq data from an

143 aggregate of 16 conditions and tissues ${ }^{44,45}$ (Supplementary Data 1, Supplementary Note 2). 
144 The predicted gene set is a notable improvement on previous annotations, both in

145 completeness and in full-length gene-level accuracy, due in part to the more complete assembly

146 (Supplementary Fig. 1, Supplementary Table 2, Supplementary Note 2). In particular, gaps

147 in the earlier genome assemblies arising from cloning biases in the Sanger sequencing process

148 and encompassing exons embedded in highly repetitive sequences have been filled by single

149 molecule long reads (Supplementary Figures 1 and 3).

151 A measure of this completeness and the utility of the $X$. tropicalis genome is provided by

152 comparing its gene set with those of vertebrate model systems with reference-quality genomes,

153 including chicken ${ }^{46}$, zebrafish ${ }^{47}$, mouse $^{48}$ and human ${ }^{49,50}$ (Supplementary Fig. 4). Notably,

154 despite the closer phylogenetic relationship between birds and mammals, $X$. tropicalis shares

155 more orthologous gene families (and mutual best hits) with human than does chicken, possibly

156 because of the loss of genomic segments in the bird lineage ${ }^{16,51}$ and/or residual incompleteness

157 of the chicken reference sequence, due to the absence of several microchromosomes ${ }^{46}$. For

158 example, of 13,009 vertebrate gene families with representation from at least four of the

159 vertebrate reference species, only 341 are missing from $X$. tropicalis versus 1,110 from chicken

160 (Supplementary Fig. 4). The current $X$. tropicalis genome assembly also resolves gene order

161 and completeness of gene structures in the long subtelomeres that were missed in previous

162 assemblies due to their highly repetitive nature (Supplementary Fig. 1F-G).

Protein-coding gene sets for other frogs

165 We annotated the new genomes of E. coqui, E. pustulosus, H. boettgeri, and $P$. adspersus 166 using transcriptome data from these species (Supplementary Data 1) and peptide homology

167 with $X$. tropicalis (Supplementary Tables 6 and 7). To include mustache toad in our cross-frog 168 comparisons we adopted the published annotation of Li et al. ${ }^{22}$ (Supplementary Note 3). We 
found 14,412 orthologous groups across the five genera with OrthoVenn $2^{52}$, including genes

170 found in at least four of the five frog genera represented (Supplementary Fig. 7B). As

171 expected, due to its reference-quality genome and well-studied transcriptome, only 72 of these

172 clusters were not represented in X. tropicalis; the other frog genomes each had between 575

173 and 712 of these genes missing (or mis-clustered), suggesting better than $95 \%$ completeness in

174 the other species. For analyses of synteny, we further restricted our attention to 7,292 one-to-

175 one gene orthologs that were present on chromosomes (as opposed to unlinked scaffolds) in

176 the "core" genomes $X$. tropicalis, H. boettgeri, E. coqui, E. pustulosus, and $P$. adspersus. The

177 total branch length in the pipanuran tree shown in Fig. 1 (including both $X$. laevis subgenomes)

178 is 2.58 substitutions per four-fold synonymous site.

\section{Repetitive landscape}

181 Centromeric and telomeric tandem repeats play a critical role in the stability of chromosome

182 structure ${ }^{53}$. Nonetheless, other kinds of repeats also play a role in the preservation of these

183 important chromosome landmarks ${ }^{54,55}$. The new $X$. tropicalis $v 10$ assembly captures sequences

184 from centromeres and distal sub-telomeres that were fragmented in the previous

185 assemblies ${ }^{18,33}$. The percentage of the genome covered by transposable elements is slightly

186 higher than previously reported ${ }^{33}$ (36.82\% vs. 34\%) (Supplementary Table 4).

188 Insertional bias in the pericentromeric regions is observed for specific families of long

189 interspersed elements (LINEs), including the relatively young Chicken Repeat 1 (CR1, ref. ${ }^{56}$ )

190 (3.14\% of the genome) and the ancient L1 (1.06\%) (Fig. 2 and Supplementary Fig. 5). The $X$.

191 tropicalis v10 assembly captures significantly more tandem repeats in the distal subtelomeric

192 portions of the genome relative to earlier assemblies. An exhaustive search for tandem repeats

193 using Tandem Repeat Finder ${ }^{57}$ determined that $10.67 \%$ of the chromosomes is covered by 
194 tandem arrays consisting of 5 or more monomeric units greater than $10 \mathrm{bp}$. Many tandem

195 repeat footprints are in gaps from previous assemblies ${ }^{18,33}$ (Supplementary Fig. 3). Our new

196 hybrid genome assembly closed many gaps containing centromeric and subtelomeric tandem

197 repeats and captured numerous subtelomeric genes (Supplementary Fig. 1). The overall

198 repeat landscape derived from the $X$. tropicalis assembly is mirrored in the other frog

199 assemblies, with similar centromeric repeats, and lengthy subtelomeres, as discussed below

200 (Supplementary Fig. 9).

201

202 Genetic variation

203 Although the $X$. tropicalis reference genotype is highly inbred, it nevertheless retains 15 long

204 heterozygous blocks ranging in size from 1.34 to $74.6 \mathrm{Mb}$. This exceeds the expectations based

205 on 17 generations of brother-sister mating, suggesting residual heterozygosity could be

206 maintained by balancing selection. Within the heterozygous blocks we observe 3.0 single

207 nucleotide variants per kilobase. To begin to develop a catalog of segregating variation we

208 sequenced pools of frogs from the Nigerian and Ivory Coast B populations, which have been

209 previously analysed using SSLP markers ${ }^{58}$. From our light pool shotgun analysis we identified a

210 total of $6,546,379$ SNPs. There were 2,482,703 variants in the Nigerian pool and 4,661,928 in

211 the Ivory Coast B pool, with 598,252 shared by both pools, pointing to substantial differentiation

212 between populations (Supplementary Fig. 6, Supplementary Note 2).

214 Evolutionary dynamics of frog chromosomes

215 Conserved synteny and ancestral chromosomes

216 Comparison of the chromosomal positions of orthologs across seven frog genomes reveals

217 extensive conservation of synteny and collinearity (Fig. 1, Supplementary Fig. 8). We identified 
219 Note 4). Each unit likely represents an ancestral pipanuran chromosome, an observation

220 consistent with the $2 n=26$ ancestral karyotype inferred from cytogenetic comparisons across

221 frogs $^{27,59}$. Over $95 \%(6,952$ of 7,292$)$ of chromosomal one-to-one gene orthologs are maintained

222 in the same unit across the five frog species, attesting to the stability of these chromosomal

223 elements (Fig. 1). The conservation of gene content per element is comparable to the $95 \%$

224 ortholog maintenance in the Muller elements in Drosophila spp. ${ }^{60}$. Despite an over two-fold

225 difference in total genome size across the sampled genomes, each ancestral pipanuran element

226 accounts for a nearly constant proportion of the total genome size, gene count, and repeat

227 count in each species, implying uniform expansions and contractions during the history of the

228 clade (Supplementary Fig. 7C).

At least some of these pipanuran elements have a deeper ancestry within amphibians.

231 Comparison with the genome of the axolotl, Ambystoma mexicanum-a member of the order

232 Caudata (salamanders and newts), and $\sim 292$ million years divergent from pipanurans ${ }^{61}$ -

233 reveals conservation of multiple syntenic units (Supplementary Fig. 8A). For example, axolotl

234 chromosomes $4,6,7$, and 14 are in near $1: 1$ correspondence with pipanuran elements $F, A, B$

235 and $\mathrm{K}$, respectively, although small pieces of $\mathrm{F}$ and $\mathrm{A}$ can be found on axolotl 10 , and parts of $\mathrm{B}$

236 can be found on axolotl 9 and 13. Other axolotl chromosomes are fusions of parts of two or

237 more pipanuran elements. For example, axolotl chromosome 5 is a fusion of a portion of $\mathrm{J}$ with

238 most of $G$; the remainder of $G$ is fused with a portion of $L$ on the $q$ arm of axolotl 2. Further

239 comparisons are needed to determine which of these rearrangements occurred on the axolotl

240 vs. the stem pipanuran lineage. Genomes from the superfamilies Leiopelmatoidea and

241 Alytoidea, which diverged prior to the radiation of pipanurans, will also be informative. 


\section{Chromosome evolution}

244 Block rearrangements of the 13 ancestral elements dominate the evolutionary dynamics of

245 pipanuran karyotypes (Table 1, Fig. 1). While element $C$ has remained intact as a single

246 chromosome across the group (except for internal inversions), all the other elements have

247 experienced translocations during pipanuran evolution. During these translocations, the

248 elements have remained intact with the exception of the breakage of elements $\mathrm{A}$ and $\mathrm{M}$ by

249 reciprocal partial arm exchange observed in P. adspersus chromosomes 3 and 6.

251 To trace the evolutionary history of centromeres shown in Fig. 1, we inferred their positions

252 using $\mathrm{HiC}$ contact map patterns as in $X$. tropicalis (where centromeres were also confirmed by

253 analysis of Cenp-a binding as described below). In general, the pericentromeres of other

254 pipanurans were characterized by the same repetitive element families found in Xenopus,

255 further corroborating their identification. Overall, we found broad pericentromeric conservation

256 among the species analyzed (Figs. 1 and 3A).

258 Robertsonian or centric translocations involving breaks and joins near centromeres account for

259 several of the rare rearrangements (Figs. 1 and 3B). For example, element G clearly

260 experienced a centric fission in the E. coqui lineage. Conversely, I and M underwent centric

261 fusion in the E. pustulosus lineage. E. coqui has experienced the most intense rearrangement,

262 including Robertsonian fissions of $A$ and $G$, a Robertsonian fusion of $\mathrm{I} / \mathrm{K}$, and a significant series

263 of Robertsonian rearrangements involving B, E, F, and $\mathrm{H}$ that resulted in Bprox/H, Bdist/Fdist,

264 and E/Fprox (Table 1, Supplementary Table 8). (Mechanistically, these "fissions" and "fusions"

265 likely occur by translocations; for a discussion see ref. ${ }^{62}$.) Elements I and $\mathrm{H}$ form the two arms of

266 a metacentric chromosome in pipids (Fig. 3A), and therefore the pipid ancestor, but are found

267 as either independent acrocentric chromosomes (e.g., in P. adspersus and L. ailaonicum) or as

268 arms of metacentrics formed by centric fusion with other elements (Supplementary Table 8). 
270 We also observed end-to-end "fusions" of metacentric chromosomes, for example, the joining of

271 D with $\mathrm{K}$ in E. pustulosus, and with element $\mathrm{E}$ in the common ancestor of pipids (Hymenochirus

272 and Xenopus) (Figs. 1 and 3C). Since bicentric chromosomes are not stably propagated

273 through mitosis, one of the two ancestral centromeres brought together by end-to-end fusion

274 must be lost or inactivated, as shown in Fig. $3 \mathrm{C}$ for the ancient D-E fusion in pipids. We note

275 that the $D$ centromere persists in both end-to-end fusions involving $D$, suggesting that

276 centromeres derived from different ancestral elements may be differentially susceptible to

277 silencing.

Using the pericentromere and subtelomere repeat landscape as a proxy, we found several examples of end-to-end chromosome fusions in which residual subtelomeric signals are preserved near the presumptive junction (Fig. 3, Supplementary Fig. 9). These include the end-to-end fusion of $X$. tropicalis-like chromosomes 9 and 10 (elements $L$ and $M$ ) to produce the Chr9_10 progenitor of $X$. laevis that is found in both the $L$ and $S$ subgenomes of this allotetraploid $^{20}$. These $X$. laevis chromosomes display evidence of decaying subtelomeric signatures in the region surrounding the ancestral L-M fusion (Fig. 1 and Supplementary Fig. 9A,B). Similarly, enrichment of subtelomerically associated repeats is observed in $H$. boettgeri 287 chromosome 8_10 (Supplementary Fig. 9C-E) near the junction between the portions of the 288 chromosome with $\mathrm{M}$ and $\mathrm{J} / \mathrm{K}$ ancestry (the $\mathrm{J} / \mathrm{K}$ fusion occurred near the base of pipids). In both cases, the centromere from element $\mathrm{M}$ (i.e., the centromere in $X$. tropicalis chromosome 9 ) is maintained after fusion. The inversion of the p-arm from Chr8S also has evidence of decaying 291 sequence but the median is less than the median JC distance at the Chr9_10 fusion, suggesting 292 that the fusion preceded the inversion. 
Rate of karyotype change

The long-range and, in most cases, chromosome-scale collinearity (Supplementary Fig. 8,

Supplementary Table 9) among the frog species we examined, despite a combined branch

297 length of 1.05 billion years (Supplementary Tables 10 and 11), parallels the synteny observed

298 in birds ${ }^{63}$ and reptiles ${ }^{64}$ but differs from the substantial chromosome variation found in

299 mammals ${ }^{15,31}$. Maintenance of collinear blocks may reflect an intrinsically slow rate of

300 rearrangement in frogs, perhaps a consequence of large regions devoid of recombination, or

301 selection favoring retention of specific gene order and chromosome structure related to

302 chromosomal functions. We inferred a total of 17 fission, fusion, translocation, and duplication

303 events (excluding smaller intra-chromosome rearrangements) resulting in a karyotype change

304 every 62 million years (Fig. 1). This rate is similar to the rate of one chromosome-number

305 change every 70 to 90 million years as previously proposed for frogs and mammals ${ }^{26,28}$ but still

306 slower than karyotype change rates for most mammals ${ }^{65}$ and many reptiles ${ }^{66}$. Of course, our

307 rate calculation is based on only seven species, and the rate may vary depending on the

308 species analyzed. Some frog taxa, such as Eleutherodactylus spp. $(2 n=16-32)$ and

309 Pristimantis spp. ${ }^{39}(2 n=22-38)$, have had a higher rate of karyotype change. On the other

310 hand, some species, such as Leptobrachium ailaonicum, L. leishanense ${ }^{14}$, and Rana

311 temporaria ${ }^{102}$, may have had no significant inter-chromosome changes over the past 205 million

312 years (Fig. 1). Nonetheless, this analysis of chromosome variation across the frog lineage

313 suggests a remarkably slow rate of karyotype evolution.

\section{Chromosome structure and conformation}

315 The stasis of Xenopus chromosomes relative to other frogs (see below) allows us to examine

316 the repetitive landscape of chromosomes that are not frequently rearranged by translocation,

317 and may be approaching a structural equilibrium. 
Centromeres, satellites, and pericentromeric repeats

320 Vertebrate centromeres are typically characterized by tandem families of centromeric satellites

321 (e.g., the alpha satellites of humans) that bind to the centromeric histone H3 protein, Cenp-a, a 322 centromere-specific variant of histone $\mathrm{H} 3^{53,67}$. Cenp-a binding satellites have been described in $323 X$ laevis $^{68}$, and here we find distantly related $X$. tropicalis satellite sequences that also co-

324 precipitate with Cenp-a. Thus, chromatin immunoprecipitation and sequencing (ChIP-seq)

325 shows that Cenp-a binding coincides with the predictions of centromere positions derived from

326 chromatin conformation analysis and repetitive content (Supplementary Fig. 5A-C,

327 Supplementary Tables 12 and 13). Importantly, this concordance supports the prediction of

328 centromere position for other species that we infer below. The Cenp-a bound-sequences are

329 arrays of $205-b p$ monomers that share a mean sequence identity greater than $95 \%$ at the

330 nucleotide level, with a specific segment of the repeating unit showing greatest variability

331 (Supplementary Fig. 10). The $X$. tropicalis centromere sequence is different from centromeric-

332 associated repeats found in $X$. laevis $^{68,69}$, suggesting the sequences evolve rapidly after

333 speciation but are maintained within the species.

All metacentric pericentromeric regions of $X$. tropicalis chromosomes are enriched in retrotransposable repetitive elements (15 Mb regions shown in Fig. 2). In other vertebrate species and Drosophila, retrotransposable elements from the pericentromeric regions are

338 involved in the recruitment of constitutive heterochromatin components ${ }^{70,71}$. Among the

339 pericentromerically enriched repeats we identified specific families belonging to LTR

340 retrotransposons (Ty3), non-LTR retrotransposons (CR1, Penelope, and L1), and DNA

341 transposable elements (PIF-Harbinger and piggyBac families) (Fig. 2, Supplementary Fig. 5).

342 CR1 (CR1-2_XT) is the most prevalent and it is among the youngest of all pericentromeric 343 retrotransposons (mean Jukes-Cantor (JC) distance to consensus of 0.05). In contrast, L1 and

344 Penelope types have a mean JC greater than 0.4 (Supplementary Fig. 5). The age of the 
repeats, indirectly measured by the JC distance, suggests that pericentromeric retrotransposons have experienced different bursts of activity and tendency to insert near the centromere.

347 Expression of active retrotransposons and random insertion can compromise chromosome 348 stability, and because silencing of these is crucial, genomes develop mechanisms to rapidly

349 silence them. Such insertions may be positively selected, and therefore amplified, to establish

350 pericentromeric heterochromatin, but may be counter selected when they insert in gene rich 351 chromosome arms.

\section{Recombination and extended subtelomeres}

354 Although meiotic recombination is distributed across chromosomes, it is enriched near the 355 chromosome ends (Supplementary Fig. 11A). While in humans, meiotic recombination is 356 suppressed close to centromeres and elevated near telomeres, recombination is still regularly 357 distributed on chromosome arms ${ }^{72}$. Other groups, including birds and fish, experience most 358 recombination events $5 \mathrm{Mb}$ away from the telomeres and only modest recombination is 359 observed outside those regions ${ }^{73-76}$. Binding events for the protein PRDM9, present in mouse, 360 rat and human, mark recombination hotspots in the chromosome arms in these species ${ }^{77}$. Given 361 that amphibians lack the prdm9 gene ${ }^{78}$, we analyzed the genomic features that colocalized in 362 areas prone to recombination.

364 We studied the distribution of recombination along $X$. tropicalis chromosomes using a previously generated Nigerian-Ivory Coast $F_{2}$ cross $^{18}$ (Supplementary Note 5, Supplementary Data 2). Half of the observed recombination is concentrated in only $160 \mathrm{Mb}(11.0 \%$ of the genome) and $36790 \%$ of the observed recombination occurs in $540 \mathrm{Mb}(37.3 \%)$. In contrast, the central regions of each chromosome are "cold", with recombination rates below 0.5 cM/Mb (Supplementary Fig. 
370 concentrated within just $30 \mathrm{Mb}$ of the ends of each chromosome and occurs only rarely

371 elsewhere (Supplementary Fig. 11A); the regions of the subtelomeres experiencing high

372 recombination are nearly 6 -fold larger than in non-amphibian genomes ${ }^{73,74}$. These rates of

373 recombination were not previously determined, since the repeat-rich subtelomeres were absent

374 from the assemblies, and markers that happened to lie in those regions showed insufficient

375 linkage to be incorporated into the maps.

377 Due to the elevated recombination, and repeat structure discussed below, we defined the

378 extended sub-telomeres as the terminal $30 \mathrm{Mb}$ of all metacentric chromosomes, and terminal 30

$379 \mathrm{Mb}$ excluding the $15 \mathrm{Mb}$ surrounding the pericentromeric regions of acrocentric chromosomes

380 (Chr3, Chr8, and Chr10) (Fig. 2). The median recombination rate in the extended subtelomeres

$381(1.73 \mathrm{cM} / \mathrm{Mb})$ is ten-fold higher than the median rate observed in the rest of the chromosome

382 arms $(0.16 \mathrm{cM} / \mathrm{Mb})$. The recombination rate in the $5-\mathrm{Mb}$ region surrounding the centromeric

383 tandem repeats is even lower $(0.04 \mathrm{cM} / \mathrm{Mb})$. Since constitutive heterochromatin in

384 pericentromeric regions is known to repress recombination, this observation is expected

385 (reviewed in refs. ${ }^{79,80}$ ). However, the centromeres of acrocentric chromosomes lie within $30 \mathrm{Mb}$

386 of the telomere, which precludes the extended sub-telomeric associated repeats (Fig. 2 and

387 Supplementary Fig. 12A).

389 We examined the relationship between rates of recombination against repetitive elements and

390 sequence motifs associated with recombination hotspots in other vertebrate species

391 (Supplementary Fig. 13A, Supplementary Table 14). Similar to chicken and zebra finch,

392 recombination is the highest in subtelomeres and positively correlates with GC content ${ }^{73,76,81}$,

393 which is consistent with GC-biased gene conversion ${ }^{82-84}$ in recombinogenic regions (median GC

$394=42.5 \%$ in the $74 \mathrm{Mb}$ in which half of the recombination occurs) vs. the non-recombinogenic

395 centers of chromosomes (median 38.8\%). As in zebra finch (Supplementary Fig. 14), 
recombination in $X$. tropicalis is strongly correlated with satellite repeats $($ Pearson correlation $=$ $\left.+0.68, R^{2}=0.457\right)$. The high density of satellite repeats (Supplementary Table 15) in highly recombinogenic subtelomeric regions suggests that unequal crossing over during meiotic recombination mediates tandem repeat expansions ${ }^{85,86}$. Notably in the extended subtelomeric regions, tandem repeats are enriched in specific tetrameric sequences (TGGG, AGGG, and

401 ACAG) compared to non-tandem repeats (Supplementary Fig. 13B). In contrast, centromeric 402 tandem repeats are completely devoid of these short sequences.

Some of the tandem arrays enriched in the terminal $30 \mathrm{Mb}$ from all chromosomes derive from portions of transposable elements such as SINE/tRNA-V, LINE/CR1, DNA/Kolobok-2

(Supplementary Fig. 12, Supplementary Table 16). For example the minisatellite expansion that arose from the family of SINE/tRNA-V present in the pipid lineage ${ }^{87}$ amplified a 52-bp portion of the 3'UTR-tail from the SINE/tRNA-V element in Xenopus tropicalis and other frog species (Supplementary Table 17). Although intact SINE/tRNA-V elements are distributed throughout the genome, the minisatellite fragment is only expanded in subtelomeric SINE/tRNA-

411 Vs, suggesting that recombination in subtelomeres has driven minisatellite expansion

412 (Supplementary Figs. 12 and 15). Interestingly, although the satellite expansions are similar in $X$. laevis and $X$. tropicalis, they differ in other frogs, suggesting that different satellite expansions can occur repeatedly during the maintenance of the long subtelomeric regions (see below).

\section{Chromatin conformation correlates with cytogenetic features}

417 To further refine our understanding of chromosome structure in $X$. tropicalis, we studied 418 chromatin conformation capture ("HiC") data from nucleated blood cells. These experiments link 419 short reads representing sequences in close three-dimensional proximity ${ }^{88}$. Fig. 4 shows 420 mapped $\mathrm{HiC}$ read pairs for chromosomes 1 and 2, with different minimum mapping quality 
421 thresholds above and below the diagonal (Fig. 4 and Supplementary Fig. 2, Supplementary

422 Note 5). We consistently observe a "wing" of intra-chromosome contacts transverse to the main

423 diagonal, which (1) intersects the main diagonal near the cytogenetically defined, Cenp-a-

424 binding centromere, and (2) indicates contacts between $p$ and q arms (Supplementary Figs. 2

425 and 16). These observations imply that interphase chromosomes are "folded" at their

426 centromeres, with contacts between distal arms. We also observe inter-chromosome contacts

427 among centromeres of different chromosomes and between their telomeres (Supplementary

428 Fig. 10A).

430 Taken together, these intra- and inter-chromosome contacts are consistent with a Rabl

431 configuration of chromosomes ${ }^{89,90}$ in Xenopus blood cells. This configuration is understood as a

432 relict structure from the previous mitosis ${ }^{91,92}$, in which the chromosomes have become

433 elongated and telomeres clustered on the nuclear membrane. Associations between

434 centromeres and between telomeres, first observed in salamander embryos ${ }^{89}$, are also

435 observed in other animals ${ }^{93,94}$, fungi ${ }^{95}$, and plants ${ }^{96-98}$. These findings suggest that remnants of

436 this 'Rabl configuration' 89 may be a common feature of post-mitotic cells across a wide range of

437 eukaryotes. Here, we quantified the degree to which chromosomes are compacted in the Rabl

438 configuration using $\mathrm{HiC}$ data and find that, with the exception of blood cell nuclei (sum of

439 squared distances (SSD) 1.465), chromosomes from early frog development (NF stages 8 to

440 23) appear more tightly constrained (mean SSD 1.384) in Rabl configuration than the more

441 specialized (liver and brain) adult tissues and sperm (mean SSD 5.583; Supplementary Fig.

442 16, Supplementary Table 18, Supplementary Note 5). Although it is possible some amount of

$443 \mathrm{HiC}$ signal may be due to residual incompleteness in the assembly and concomitant

444 mismapping of reads to repeat sequences, these observations are robust to quality filtering,

445 even when using single-copy sequences. Furthermore, such contacts are weakest in sperm ${ }^{16}$, a 
control that argues strongly against sequence mismapping artefacts (Supplementary Fig. 10B,

447 Supplementary Note 5).

448

449 We also observed three-dimensional associations between pericentromeric regions of different 450 chromosomes, based on enriched HiC contacts ${ }^{90,99}$ (Fig. 4). As with the Rabl signal, these

451 "contacts" are accentuated when HiC reads are allowed to map permissively (Methods), which

452 suggests that they may be influenced by common repetitive pericentromeric sequences shared

453 among chromosomes. The signal persists in weaker form with more stringent read mapping,

454 however, and either represents bona fide signal or residual incompleteness of the

455 pericentromeric assembly. Notably, the correlation between centromere position and the

456 observed intra-chromosome folding and inter-chromosome contacts at centromeres allows us to

457 use $\mathrm{HiC}$ analysis and principal component analysis (PCA) of intra- and inter-chromosome

458 contacts $^{97}$ to infer the likely centromeric positions based purely on $\mathrm{HiC}$ data in frogs whose

459 cytogenetics are less well-studied (see below).

\section{Chromatin compartments}

462 Chromatin contacts in human $88,100,101$, mouse ${ }^{101}$, chicken ${ }^{102}$ and other phylogenetically diverse

463 species ${ }^{103-105}$ often show a characteristic checkerboard pattern that is superimposed on the

464 predominant near-diagonal signal. This pattern implies an alternating 'A/B' compartment

465 structure with enriched intra-compartment contacts within chromosomes (Fig. 5A), which has

466 been linked with G-banding in humans ${ }^{106}$. X. tropicalis also exhibits an A/B compartment

467 pattern, which emerges as alternating gene rich ('A') and gene poor ('B') regions (median 19.99

468 genes/Mb in $\mathrm{A}$ and 9.99 genes/Mb in B) (Fig. 5B). A/B compartments are also differentiated by

469 repetitive content ${ }^{101}$, with A-compartment domains showing slight enrichment (1.21-1.44 fold) in

470 DNA transposons of the DNA/Kolobok-T2, DNA/hAT-Charlie, and Mariner-Tc1 families. B- 
471 compartment domains had significantly higher enrichment for DNA transposons (DNA/hAT-Ac,

472 Mar-Tigger) and retrotransposons (Ty3/metaviridae and CR1), among other repeats (1.12-2.11

473 fold) (Fig. 5C). The association between repeats overrepresented in A and B compartments is

474 also captured in one of the principal components obtained from the repeat densities of all

475 chromosomes (Supplementary Note 5); we detect a modest negative correlation $(R=-0.44)$

476 between the $\mathrm{HiC}$ eigenvectors that classified A/B compartments and the third principal

477 component eigenvectors obtained from the repeat density matrix (Supplementary Fig. 5B).

478 The association between chromatin condensation and repeat type could be due to a preference

479 for certain transposable elements to insert in specific chromatin contexts, or chromatin

480 condensation to be controlled in part by transposable element content, or a combination of

481 these factors. However, we were unable to find any correlation of $A B$ compartments with the G-

482 banding of condensed chromosomes in X. tropicalis ${ }^{107,108}$.

Higher-order interactions

485 Chromatin conformation contacts also provide clues to the organization of chromosomes within

486 the nucleus. We observe non-random $\left(\chi^{2}(81, n=24,987,749)=3,049,787 ; p<2.2 \times 10^{-308}\right)$

487 associations between chromosomes in blood cell nuclei (Fig. 4B, Supplementary Tables 19

488 and 20): (a) chromosome 1 is enriched for contacts with chromosomes 2-8 (mean 1.05x

489 enrichment), and depleted of contacts with 9 and 10 (mean 0.89x); (b) among themselves,

490 chromosomes 2-8 show differential contact enrichment or depletion; and (c) chromosomes 9

491 and 10 are enriched $(1.17 \times)$ for contacts with one another but are depleted with respect to all

492 other chromosomes. These observations suggest the presence of distinct chromosome

493 territories ${ }^{89,109-111}$, where chromosomes 2-8 localized more proximal to-and arrayed around-

494 chromosome 1, with chromosomes 9 and 10 relatively sequestered from chromosome 1 (Fig.

495 4C). The contact enrichment between chromosomes 9 and 10 is particularly notable because 
these short chromosomes ( 91.2 and $52.4 \mathrm{Mb}$, respectively) have become fused in the $X$. laevis

497 lineage ${ }^{112}$, which might have been enabled by their persistent nuclear proximity ${ }^{113}$.

Between chromosomes, $p-p$ and $q-q$ arm interactions exhibit a small but significant enrichment

$500\left(1.059 \times\right.$ enrichment; $\left.\chi^{2}(1, n=24,786,496)=17,037 ; p<2.2 \times 10^{-308}\right)$ over $p-q$ arm contacts. This

501 is a general feature of metacentric and sub-metacentric chromosomes also observed in other

502 frog genomes (see below), except E. coqui $\left(0.928 \times\right.$ enrichment; $\chi^{2}(1, n=6,850,547)=3,914 ; p$

$503<2.2 \times 10^{-308}$ ), the chromosomes of which are predominantly acrocentric. Finally, the p-arms of

504 chromosomes 3, 4, 8, and 9 are enriched for contacts with chromosome 10, with the sub-

505 metacentric chromosomes 3 and 8 showing the strongest enrichment (and a slight preference

506 between p-arms). The q-arms of chromosomes 3 and 8, however, exhibit a slight enrichment for

507 contacts with chromosomes 1, 2, 4, and 5. Taken together, these observations suggest

508 colocalization of the $p$ and $q$ arms of chromosomes 3 and 8 in blood cell nuclei.

\section{Conclusions}

511 Anuran amphibians play a central role in biology, not simply as a globally distributed animal

512 taxon, but also as key subjects for research in areas that range from ecology and evolution to

513 cell and developmental biology. The genomic resources generated here will thus provide

514 important tools for further studies. Given the crucial role of $X$. tropicalis for genomic analysis of

515 development and regeneration ${ }^{114,115}$, the improvements to our understanding of its genome

516 reported here will provide a more finely grained view of biomedically important genetic and

517 epigenetic mechanisms. This new genome is also important from the standpoint of evolutionary

518 genomics, as comparisons between the genomes of $X$. tropicalis and $X$. laevis shed light on

519 mechanisms of genome duplication ${ }^{115}$. The new genome described here for $H$. boettgeri,

520 another pipid frog, is also significant in this regard, as it enables an interesting comparison of 
521 Xenopus genomes to that of a closely related outgroup. Moreover, the genomes of E. coqui and

522 E. pustulosus provide a foundation for future studies of the evolution of ontogenies and of their

523 underlying developmental mechanisms, as E. coqui is a direct-developing frog with no tadpole

524 stage ${ }^{116}$ and E. pustulosus, a foam-nesting frog, is a model for studying mating calls and female

525 mate choice ${ }^{116}$. In addition to their interesting life histories, both frogs display interesting

526 patterns of gastrulation ${ }^{117,118}$. Finally, recent work has demonstrated the efficacy of genetic or

527 genomic analysis for understanding the impact of chytrid fungus on various amphibian

528 species ${ }^{119}$. A deeper and broader understanding of amphibian genomes will be useful in the

529 context of the global decline of amphibian populations ${ }^{120,121}$.

532 Genomic extraction and sequencing.

533 High molecular weight DNA was extracted from blood of an $\mathrm{F}_{17}$ Xenopus tropicalis Nigerian

534 strain female (ref. ${ }^{18}$; Supplementary Note 1). Paired-end (PE) Illumina shotgun libraries were

535 constructed by the QB3 Functional Genomics Laboratory (FGL) and sequenced on an Illumina

536 HiSeq 2500 as $2 \times 250$ bp reads at the Vincent J. Coates Genomics Sequencing Lab (VCGSL) at

537 the University of California, Berkeley. Single-molecule real-time (SMRT) continuous long-read

538 (CLR) sequencing was performed at the HudsonAlpha Institute for Biotechnology on PacBio

539 RSII machines with P6-C4 chemistry (Supplementary Note 1, Supplementary Data 1). 10x

540 Genomics Chromium linked-read sequencing was carried out at HudsonAlpha on HiSeq XTen

541 (Supplementary Note 1).

543 Xenopus tropicalis genome assembly and annotation.

544 Chromium linked reads (10x Genomics) were assembled with Supernova ${ }^{122}(\mathrm{v} 1.1 .5)$. This

545 assembly was used to seed the assembly of continuous long reads (PacBio) using DBG2OLC ${ }^{123}$ 
546 (commit 1f7e752). An independent PacBio-only assembly was constructed with Canu ${ }^{124}$ (v1.6-

547 132-gf9284f8). These two assemblies were combined, or metassembled, using MUMmer ${ }^{125}$

548 (v3.23) and quickmerge ${ }^{126}$ commit e4ea490 (Supplementary Fig. 1A, Supplementary Note 2).

549 Residual haplotypic redundancy was identified and removed (Supplementary Fig. 1B,

550 Supplementary Note 2). The non-redundant metassembly was scaffolded with Sanger paired-

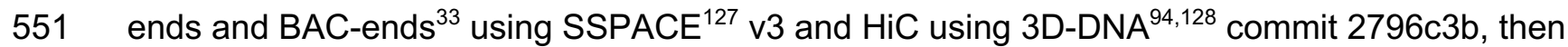

552 manually curated in JuiceBox ${ }^{129,130}$ v1.9.0 (Supplementary Note 2). The assembly was

553 polished with Arrow ${ }^{131}$, Pilon $^{132}$ v1.23, and then FreeBayes ${ }^{133}$ (v1.1.0-54-g49413aa) with ILEC

554 (map4cns; https://bitbucket.org/rokhsar-lab/map4cns). The genome was annotated with the

555 DOE-JGI Integrated Gene Call pipeline ${ }^{134}$ (IGC) using transcript assemblies (TAs) generated

556 with Trinity ${ }^{135,136}$ v2.5.1 from multiple developmental stages and tissues (Supplementary Data

557 1, Supplementary Note 2). RepeatModeler ${ }^{137}$ v1.0.11 was run on all frog species. The frog and

558 ancestral repeat libraries from RepBase ${ }^{138}$ v23.12 were combined with the repeats consensuses

559 identified by Repeat Modeler. The merged repeat library was used to annotate repeats of all

560 frogs with RepeatMasker ${ }^{139}$ v4.0.7 (Supplementary Notes 2 and 3).

Hymenochirus boettgeri metaphase chromosome spread.

563 Stage 26 tadpoles $(n=10)$ were incubated at room temperature in $0.01 \%$ colchicine and $1 \times$

564 MMR for 4-6 hr. After removing the yolky ventral portion of the tadpoles, the remaining dorsal

565 portions were pooled together in deionized water and allowed to stand for $20 \mathrm{~min}$. The dorsal

566 portions were transferred to $0.2 \mathrm{~mL}$ of $60 \%$ acetic acid in deionized water and allowed to stand

567 for $5 \mathrm{~min}$. The tissue was then pipetted onto a positively charged microscope slide, and excess

568 acetic acid was blotted away. To flatten the tissue and promote chromosome spreading, the

569 slide was covered with a coverslip and a lead brick was placed on top of it for $5 \mathrm{~min}$. The slide

570 and coverslip were then placed on dry ice for $5 \mathrm{~min}$. The coverslip was removed from the frozen

571 slide, and the slide was stained with $0.1 \mathrm{mg} / \mathrm{mL}$ Hoechst Stain solution for $5 \mathrm{~min}$. A fresh 
572 coverslip was then mounted on the slide using VectaShield, and the edges were sealed with nail

573 polish. Chromosomes in metaphase spreads (Supplementary Fig. 7A) were imaged on an

574 Olympus BX51 Fluorescence Microscope run with Metamorph software using a 60× oil

575 objective. Chromosome number was counted in 75 separate metaphase spreads.

577 Genome and transcriptome sequencing of five pipanurans.

578 Illumina PE 10x Genomics Chromium linked-read whole-genome libraries for E. pustulosus

579 (from liver), E. coqui (from blood) and H. boettgeri (from liver) were sequenced on an HiSeq X at

580 the HudsonAlpha Institute for Biotechnology. PacBio SMRT Sequel I CLR data were generated

581 at UC Davis DNA Technologies and Expression Analysis Core for each of E. pustulosus and $H$.

582 boettgeri from liver samples. In addition, two TruSeq Illumina PE libraries (from kidney) and two

583 Nextera mate-pair libs (from liver) for E. coqui were prepared. HiC chromatin conformation

584 capture libraries were prepared for H. boettgeri, E. pustulosus, and E. coqui using the

585 Dovetail ${ }^{\mathrm{TM}} \mathrm{HiC}$ Kit for Illumina following the "Animal Tissue Samples" protocol. HiC libraries

586 were sequenced on the Illumina HiSeq 4000 by the VCGSL.

588 Illumina TruSeq Stranded mRNA Library Prep Kit libraries were prepared from E. pustulosus 589 stages 45 and 56 whole tadpoles (gut excluded) and various adult tissues dissected from frogs 590 maintained at the University of the Pacific. Brain $(n=3)$, dorsal skin $(n=2)$, eggs $(n=2)$, eye $(n$ $591=2)$, heart $(n=2)$, intestine $(n=2)$, larynx $(n=3)$, liver $(n=2)$, lung $(n=2)$, and ventral skin $(n=$ 592 2) samples were washed twice with PBS, homogenized in TRIzol Reagent, and centrifuged,

593 followed by flash freezing of the supernatant. RNA was isolated following the TR/zol Reagent

594 User Guide (Pub. No. MAN0001271 Rev. A.0) protocol. In addition, H. boettgeri eggs were 595 homogenized in TRIzol Reagent and processed according to manufacturer's instructions. RNA 596 was then isolated using the QIAGEN RNeasy Mini Kit (cat 74104). An Illumina mRNA library 597 was prepared using the Takara PrepX RNA-Seq for Illumina Library Kit by the Functional 
598 Genomics Laboratory at the University of California Berkeley. All libraries were sequenced at

599 the VCGSL on an HiSeq 4000 as 151 bp PE reads. See Supplementary Note 3 for additional

600 details about DNA/RNA extractions and library preparations, and Supplementary Data 1 for a

601 complete list of DNA/RNA sequencing data generated for E. coqui, E. pustulosus, and $H$.

602 boettgeri.

603

604 Assembly and annotation of five pipanuran genomes.

605 Contigs were assembled with Supernova ${ }^{122}$ v2.0.1 (E. pustulosus and H. boettgeri) or

606 Meraculous ${ }^{140,141}$ v2.2.4 (E. coqui). For E. coqui, residual haplotypic redundancy was removed

607 using custom scripts (https://github.com/abmudd/Assembly) prior to scaffolding with SSPACE ${ }^{127}$

608 (v3.0). E. pustulosus and H. boettgeri contigs were ordered and oriented using MUMmer ${ }^{125}$

609 (v3.23) alignments to PBEC-polished (map4cns commit dd89f52; https://bitbucket.org/rokhsar-

610 lab/map4cns) DBG2OLC ${ }^{123}$ (commit 1f7e752) hybrid contigs (Supplementary Note 3). All three

611 assemblies were scaffolded further with linked-reads and Scaff10X (v2.1;

612 https://sourceforge.net/projects/phusion2/files/scaff10x).

614 E. pustulosus and $H$. boettgeri chromosome-scale scaffolds were constructed with Dovetail

615 Genomics $\mathrm{HiC}$ via the HiRise scaffolder ${ }^{142}$, followed by manual curation in JuiceBox ${ }^{128-130}$

616 v1.9.0. Due to the fragmented nature of the E. coqui assembly, initial chromosome-scale

617 scaffolds were first constructed by synteny with E. pustulosus, then refined in JuiceBox ${ }^{128-130}$

618 v1.9.0. Gaps in the E. pustulosus and H. boettgeri assemblies bridged by PacBio reads were

619 resized using custom scripts (pbGapLen; https://bitbucket.org/bredeson/artisanal) and filled with

620 PBJelly ${ }^{143}$ (PBSuite v15.8.24). These two assemblies were polished with FreeBayes and ILEC

621 (map4cns commit dd89f52; https://bitbucket.org/rokhsar-lab/map4cns). A final round of gap-

622 filling was then performed on the three assemblies using Platanus ${ }^{144}$ (v1.2.1). 
624 Previously published L. ailaonicum ${ }^{23}$ (GCA_018994145.1) and P. adspersus ${ }^{21}$

625 (GCA_004786255.1) assemblies were manually corrected in JuiceBox ${ }^{128-130}$ (v1.11.08) using

626 their respective $\mathrm{HiC}$ and Chicago data (Supplementary Data 1). Gaps in the corrected $P$.

627 adspersus scaffolds were resized with PacBio reads (as described above) and filled using

628 Platanus $^{144}$ (v1.2.1) with published TruSeq Illumina data obtained from NCBI (PRJNA439445).

629 As described elsewhere ${ }^{145}$, all assemblies were screened for contaminants prior to scaffolding,

630 and only final scaffolds and contigs longer than $1 \mathrm{~kb}$ were retained for downstream analyses.

631 More details on assembly procedures can be found in (Supplementary Note 3).

Genomic repeats in all five species were annotated with RepeatMasker ${ }^{137,139}$ (v4.0.7 and v4.0.9) using the repeat library generated above. Protein-coding genes were annotated for E. coqui, E. pustulosus, $H$. boettgeri, and $P$. adspersus using the IGC ${ }^{134}$ pipeline with homology and transcript evidence. For each respective species, newly generated RNA-seq data were

637 combined with public H. boettgeri ${ }^{20}$ (BioProject PRJNA306175) and P. adspersus ${ }^{21}$ (BioProject

638 PRJNA439445) data, and unpublished E. coqui data (stages 7, 10, and 13 hindlimb [Harvard 639 University]; stage 9-10 tail fin skin [French National Center for Scientific Research]). Transcript assemblies used as input to IGC were assembled with Trinity ${ }^{135,136}$ (v2.5.1) and filtered using the

641 heuristics described in Supplementary Note 3.

\section{Synteny and ancestral chromosome inference.}

644 One-to-one gene ortholog set between frog proteomes was obtained from the output from

645 OrthoVenn2 ${ }^{52}$ using an E-value of $1 \times 10^{-5}$ and an inflation value of 1.5 (Supplementary Note 4).

646 The assemblies of all frog species and axolotl were pairwise aligned against the $X$. tropicalis

647 genome using cactus $^{146}$ (commit e4d0859) (Supplementary Note 4). Pairwise collinearity runs

648 were merged into runs of collinearity with ROAST/MULTIZ ${ }^{147}$ (v012109) using the phylogenetic

649 topology from TimeTree ${ }^{148}$ and sorted with last ${ }^{149}$ (v979) (Supplementary Note 4). 
651 Phylogeny and estimation of sequence divergence.

652 Fourfold degenerate bases of one-to-one orthologs were obtained and reformatted from the

653 MAFFT alignment as described in Mudd et al. ${ }^{145}$ (Supplementary Note 4). The maximum-

654 likelihood phylogeny was obtained with $\mathrm{RAxML}^{150}$ (v8.2.11) using the GTR+Gamma model of

655 substitution with outgroup Ambystoma mexicanum. Divergence times were calculated with

656 MEGA7 ${ }^{151}$ (v7.0.26) with the GTR+Gamma model of substitution using Reltime method ${ }^{152}$.

658 Chromosome evolution.

659 Customized scripts ${ }^{145}$ were used to extract pairwise alignments from the ROAST-merged MAF

660 file and converted into runs of collinearity. The runs of collinearity were visualized with Circos ${ }^{153}$

661 (v0.69-6) (Supplementary Note 4).

662

663 Centromeres, satellites, and pericentromeric repeats.

664 Tandem repeats were called using Tandem Repeat Finder7 (trf genome.fa 2578010502000

$665-$ - 6 -d -ngs). To identify tandem repeats enriched in pericentromeric and subtelomeric regions,

666 we extracted the monomer sequences of all tandem repeats overlapping the region of interest.

667 A database of non-redundant monomers was created by making a dimer database. Dimers

668 were clustered with BlastClust ${ }^{154}$ v2.2.26 (-S 75 -p F -L 0.45 -b F -W 10). A non-redundant

669 monomer database was created using the most common monomer size from each cluster. The

670 non-redundant sequences were mapped to the genome with BLASTN ${ }^{155}$ (-outfmt 6 -evalue

671 1e3). The enriched monomeric sequences in centromeres and subtelomeres were identified by

672 selecting the highest normalized rations of tandem sequence footprints in the region of interest

673 over the remaining portions of the genome. For more detail, see Supplementary Note 5.

674 


\section{Genetic variation.}

676 Reads were aligned with BWA-MEM ${ }^{156}$ (v0.7.17-r1188) and alignments were processed using

677 SAMtools ${ }^{157}$ (v1.9-93-g0ca96a4), keeping only properly paired reads (samtools view -f3 -F3852)

678 for variant calling. Variants were called with FreeBayes ${ }^{133}$ (v1.1.0-54-g49413aa; --standard-

679 filters --genotype-qualities --strict-vcf --report-monomorphic). Only bi-allelic SNPs with depth

680 within mode \pm 1.78 SDs were retained. An allele-balance filter [0.3-0.7] for heterozygous

681 genotypes was also applied. Segmental heterozygosity/homozygosity were estimated using

682 windows of $500 \mathrm{~kb}$ with 50-kb step using BEDtools ${ }^{158}$ (v2.28.0) for pooled samples or snvrate ${ }^{159}$

683 (v2.0; https://bitbucket.org/rokhsar-lab/wgs-analysis). For more detail, see Supplementary

$684 \quad$ Note 2.

\section{GC-content, gene, and repeat landscape.}

687 GC-content percentages were calculated in 1-Mb bins sliding every $50 \mathrm{~kb}$. Gene densities were obtained using a window size of $250 \mathrm{~kb}$ sliding every $12.5 \mathrm{~kb}$. The repeat density matrix for $X$. tropicalis was obtained by counting base pairs per $1 \mathrm{Mb}$ (sliding every $200 \mathrm{~kb}$ ) covered by repeat families and classes of repeats. The principal component analysis (PCA) was performed on the density matrix composed of 7,253 1-Mb bins and 3,070 repeats (Supplementary Note 5). The first (PC1) and second (PC2) components were smoothed using a cubic spline method.

\section{Chromatin immunoprecipitation.}

695 Xenopus tropicalis XTN-6 cells (Gorbsky and Horb, unpublished) were grown in 70\% calcium-

696 free L-15 (US Biologicals cat\# L2101-02-50L), pH 7.2/10\% Fetal Bovine Serum/Penicillin-

697 Streptomycin (Invitrogen cat\# 15140-163) at RT. Native MNase ChIP-seq protocol performed as

698 described previously in Smith et al. ${ }^{69}$. Approximately 40 million cells were trypsinized and

699 collected; nuclei were isolated by dounce extraction and collected with a sucrose cushion.

700 Chromatin was digested to mononucleosomes by MNase. Nuclei were lysed and soluble 
nucleosomes were extracted overnight at $4{ }^{\circ} \mathrm{C}$. Extracted mononucleosomes were precleared with Protein A dynabeads (Invitrogen cat\# 100-02D) for at least $4 \mathrm{~h}$ at $4{ }^{\circ} \mathrm{C}$. A sample was taken

703 for input after preclearing. Protein A dynabeads were bound to $10-\mu \mathrm{g}$ antibody (either Rb-anti-

704 XI Cenp-a, cross-reactive with X. tropicalis, Rb-anti-H4 Abcam cat\# 7311 or Rb-anti-H3 Abcam

705 cat\# 1791) and incubated overnight with precleared soluble mononucleosomes at $4{ }^{\circ} \mathrm{C}$.

706 Dynabeads bound to Rabbit IgG antibody (Jackson ImmunoResearch cat\#011-000-003) were

707 collected with a magnet and washed three times with TBST (0.1\% Triton X-100) before elution

708 with $0.1 \%$ SDS in TE and proteinase $\mathrm{K}$ incubation at $65^{\circ} \mathrm{C}$ with shaking for at least $4 \mathrm{~h}$. Isolated

709 and input mononucleosomes were size-selected using Ampure beads (Beckman cat\# A63880)

710 and prepared for sequencing using the NEBNext ultra ii DNA library prep kit for Illumina (NEB

711 cat\# E7654). Three replicates were sequenced on an Illumina HiSeq 4000 lane $2 \times 150$ bp by the

712 Stanford Functional Genomics Facility. PE reads were trimmed with Trimmomatic ${ }^{160}$ v0.39

713 filtering for universal Illumina primers and for Nextera-PE indices. Processed PE reads were

714 mapped with minimap2 (ref. ${ }^{161}$ ) v2.17-r941 against the unmasked genome reference.

715 Samtools ${ }^{157}$ v1.9 was used for sorting and indexing the alignment. Read counts (MapQ0) per

716 10-kb bin (non-overlapping) for all samples were calculated with multiBamSummary from

717 deeptools ${ }^{162}$ v3.3.0. Read counts were normalized by the total number of counts in the

718 chromosomes per sample (Supplementary Note 5).

720 Recombination and extended subtelomeres.

721 The reads from the $F_{2}$ mapping population ${ }^{18}$ were aligned to the $v 10$ genome using BWA-

$722 \operatorname{MEM}^{156}$ (v0.7.17-r1188). Variants were called using FreeBayes ${ }^{133}$ (v1.1.0-54-g49413aa; --

723 standard-filters --genotype-qualities --strict-vcf). SNPs were filtered, and valid $F_{2}$ mapping sites

724 were selected when the genotypes of the Nigerian F0 and the ICB $F_{0}$ were fixed and different

725 and there was a depth of at least 10 for each $F_{0}$ SNP. Maps were calculated using JoinMap ${ }^{163}$

726 v4.1 (Supplementary Note 5, Supplementary Data 2). The variation on the linkage map was 
727 smoothed using the cubic spline function calculated every $500 \mathrm{~kb}$. The Pearson correlation

728 coefficient was calculated between recombination rates and genomic features that include GC

729 content, repeat densities, and densities of reported CTCF and recombination hotspots ${ }^{164,165}$.

731 Chromatin conformations and higher-order interactions.

$732 \mathrm{HiC}$ read pairs were mapped with Juicer $^{128}$ (v1.5.6) and observed counts were extracted at $1 \mathrm{Mb}$

733 resolution with JuicerTools. Centromeres were estimated manually in JuiceBox ${ }^{129}$ and refined

734 with Centurion ${ }^{99}$ v0.1.0-3-g985439c using ICE-balanced MapQ0 matrices

735 (https://bitbucket.org/rokhsar-lab/xentr10/src/master/hic). Rabl chromatin structure was visualized

736 with PCA from Knight-Ruiz ${ }^{166}$-balanced MapQ30 matrices, and significance estimated by

737 permutation testing using custom $\mathrm{R}$ scripts. Rabl constraint between $\mathrm{p}$ - and q-arms was

738 measured as the sum of square distances (SSD) in PC1-PC2 dimensions, calculated between

739 non-overlapping bins traveling sequentially away from the centromere. Inter-/intra-chromosomal

740 contact enrichment analyses were quantified from MapQ30 matrices using $\chi^{2}$ tests in $\mathrm{R}^{167} \mathrm{v} 3.5 .0$

741 (Supplementary Note 5; https://bitbucket.org/rokhsar-lab/xentr10/src/master/hic).

743 A/B compartments.

744 A/B compartments were called with custom $\mathrm{R}^{167}$ (v3.5.0) scripts from Knight-Ruiz-balanced

745 (observed / expected normalized) MapQ30 HiC contact correlation matrices generated with

746 Juicer ${ }^{128}$ (Supplementary Note 5). Pearson's correlation between eigenvectors of PC1 from the

$747 \mathrm{HiC}$ correlation matrix and gene density were used to designate $\mathrm{A}$ and $\mathrm{B}$ compartments per

748 chromosome. 


\section{Data Availability}

751 The assemblies, annotations, and raw data are deposited in NCBI for v10 X. tropicalis

752 (BioProjects PRJNA577946 and PRJNA726269), E. coqui (BioProject PRJNA578591), E.

753 pustulosus (BioProject PRJNA578590), H. boettgeri (BioProject PRJNA578589), L. ailaonicum

754 (BioProject PRJNA578588), and P. adspersus (BioProject PRJNA578592).

755

756

\section{Code Availability}

757 All custom scripts used in this work can be found at https://bitbucket.org/rokhsar-lab/xentr10 and

758 https://github.com/abmudd/Assembly.

759

\section{References}

761 1. Cannatella, D. C. \& de Sá, R. O. Xenopus laevis as a model organism. Syst. Biol. 42, 476$762507(1993)$.

763 2. Beetschen, J. C. How did urodele embryos come into prominence as a model system? Int.

$764 \quad$ J. Dev. Biol. 40, 629-636 (1996).

765 3. Brown, D. D. A tribute to the Xenopus laevis oocyte and egg. J. Biol. Chem. 279, 4529176645299 (2004).

767 4. Harland, R. M. \& Grainger, R. M. Xenopus research: metamorphosed by genetics and 768 genomics. Trends Genet. 27, 507-515 (2011).

769 5. Gurdon, J. B. \& Hopwood, N. The introduction of Xenopus laevis into developmental 770 biology: of empire, pregnancy testing and ribosomal genes. Int. J. Dev. Biol. 44, 43-50 $771 \quad$ (2000).

772 6. Blaustein, A. R. \& Dobson, A. A message from the frogs. Nature 439, 143-144 (2006). 
773 7. Farrer, R. A. et al. Multiple emergences of genetically diverse amphibian-infecting chytrids include a globalized hypervirulent recombinant lineage. Proc. Natl. Acad. Sci. U. S. A. 108, 18732-18736 (2011).

8. Whiles, M. R. et al. Disease-driven amphibian declines alter ecosystem processes in a tropical stream. Ecosystems 16, 146-157 (2013).

9. Gomes, A. et al. Bioactive molecules from amphibian skin: their biological activities with reference to therapeutic potentials for possible drug development. Indian J. Exp. Biol. 45, 579-593 (2007).

10. McCallum, M. L. Amphibian decline or extinction? Current declines dwarf background

11. Ryan, M. J., Fox, J. H., Wilczynski, W. \& Rand, A. S. Sexual selection for sensory exploitation in the frog Physalaemus pustulosus. Nature 343, 66-67 (1990).

12. Romero-Carvajal, A. et al. Embryogenesis and laboratory maintenance of the foam-nesting túngara frogs, genus Engystomops (= Physalaemus). Dev. Dyn. 238, 1444-1454 (2009).

13. Miller, K. E., Session, A. M. \& Heald, R. Kif2a scales meiotic spindle size in Hymenochirus boettgeri. Curr. Biol. 29, 3720-3727.e5 (2019).

14. Minsuk, S. B. \& Keller, R. E. Surface mesoderm in Xenopus: a revision of the stage 10 fate

15. Ferguson-Smith, M. A. \& Trifonov, V. Mammalian karyotype evolution. Nat. Rev. Genet. 8,

16. Zhang, G. et al. Comparative genomics reveals insights into avian genome evolution and adaptation. Science 346, 1311-1320 (2014).

17. Kiazim, L. G. et al. Comparative mapping of the macrochromosomes of eight avian species provides further insight into their phylogenetic relationships and avian karyotype evolution. Cells 10, (2021).

18. Mitros, T. et al. A chromosome-scale genome assembly and dense genetic map for 
Xenopus tropicalis. Dev. Biol. 452, 8-20 (2019).

800 19. Niu, L. et al. Three-dimensional folding dynamics of the Xenopus tropicalis genome. Nat. Genet. 53, 1075-1087 (2021).

20. Session, A. M. et al. Genome evolution in the allotetraploid frog Xenopus laevis. Nature 538, 336-343 (2016).

21. Denton, R. D., Kudra, R. S., Malcom, J. W., Du Preez, L. \& Malone, J. H. The African Bullfrog (Pyxicephalus adspersus) genome unites the two ancestral ingredients for making

22. Li, J. et al. Genomic and transcriptomic insights into molecular basis of sexually dimorphic nuptial spines in Leptobrachium leishanense. Nat. Commun. 10, 5551 (2019).

23. Li, Y. et al. Chromosome-level assembly of the mustache toad genome using thirdgeneration DNA sequencing and Hi-C analysis. Gigascience 8, (2019).

24. Lu, B. et al. A large genome with chromosome-scale assembly sheds light on the evolutionary success of a true toad (Bufo gargarizans). Mol. Ecol. Resour. 21, 1256-1273

25. Sun, Y.-B., Zhang, Y. \& Wang, K. Perspectives on studying molecular adaptations of

26. Wilson, A. C., Sarich, V. M. \& Maxson, L. R. The importance of gene rearrangement in evolution: evidence from studies on rates of chromosomal, protein, and anatomical evolution. Proc. Natl. Acad. Sci. U. S. A. 71, 3028-3030 (1974).

27. Morescalchi, A. Evolution and karyology of the amphibians. Boll. Zool. 47, 113-126 (1980).

28. Bush, G. L., Case, S. M., Wilson, A. C. \& Patton, J. L. Rapid speciation and chromosomal evolution in mammals. Proc. Natl. Acad. Sci. U. S. A. 74, 3942-3946 (1977). regulators. Nature 554, 50-55 (2018). 
825

826

827

828

829

830

831

832

833

834

835

836

837

838

839

840

841

842

843

844

845

846

847

848

849

850

30. Smith, J. J. et al. A chromosome-scale assembly of the axolotl genome. Genome Res. 29, $317-324$ (2019).

31. Deakin, J. E., Graves, J. A. M. \& Rens, W. The evolution of marsupial and monotreme chromosomes. Cytogenet. Genome Res. 137, 113-129 (2012).

32. Bogart, J. P., Balon, E. K. \& Bruton, M. N. The chromosomes of the living coelacanth and their remarkable similarity to those of one of the most ancient frogs. J. Hered. 85, 322-325 (1994).

33. Hellsten, U. et al. The genome of the Western clawed frog Xenopus tropicalis. Science 328, 633-636 (2010).

34. Carneiro, M. O. et al. Pacific biosciences sequencing technology for genotyping and variation discovery in human data. BMC Genomics 13, 375 (2012).

35. Koren, S. et al. Hybrid error correction and de novo assembly of single-molecule sequencing reads. Nat. Biotechnol. 30, 693-700 (2012).

36. Quail, M. A. et al. A tale of three next generation sequencing platforms: comparison of lon Torrent, Pacific Biosciences and Illumina MiSeq sequencers. BMC Genomics 13, 341 (2012).

37. Loomis, E. W. et al. Sequencing the unsequenceable: Expanded CGG-repeat alleles of the fragile X gene. Genome Research vol. 23 121-128 (2013).

38. Feng, Y.-J. et al. Phylogenomics reveals rapid, simultaneous diversification of three major clades of Gondwanan frogs at the Cretaceous-Paleogene boundary. Proc. Natl. Acad. Sci. U. S. A. 114, E5864-E5870 (2017).

39. Schmid, M. et al. The chromosomes of Terraranan frogs. Insights into vertebrate cytogenetics. Cytogenetic and Genome Research vols 130-131 1-14 (2010).

40. Rabello, M. N. Chromosomal studies in Brazilian anurans. Caryologia 23, 45-59 (1970).

41. Scheel, J. J. The chromosomes of some African anuran species. in Genetics and Mutagenesis of Fish 113-116 (Springer Berlin Heidelberg, 1973). doi:10.1007/978-3-642- 
65700-9_11.

42. Mezzasalma, M., Glaw, F., Odierna, G., Petraccioli, A. \& Guarino, F. M. Karyological analyses of Pseudhymenochirus merlini and Hymenochirus boettgeri provide new insights into the chromosome evolution in the anuran family Pipidae. Zoologischer Anzeiger - A Journal of Comparative Zoology 258, 47-53 (2015).

43. Temple, G. et al. The completion of the mammalian gene collection (MGC). Genome Res. 19, 2324-2333 (2009).

44. Marin, R. et al. Convergent origination of a Drosophila-like dosage compensation mechanism in a reptile lineage. Genome Research vol. 27 1974-1987 (2017).

45. Owens, N. D. L. et al. Measuring absolute RNA copy numbers at high temporal resolution reveals transcriptome kinetics in development. Cell Reports vol. 14 632-647 (2016).

46. Warren, W. C. et al. A new chicken genome assembly provides insight into avian genome structure. G3: Genes|Genomes|Genetics vol. 7 109-117 (2017).

47. Howe, K. et al. The zebrafish reference genome sequence and its relationship to the human genome. Nature 496, 498-503 (2013).

48. Mouse Genome Sequencing Consortium et al. Initial sequencing and comparative analysis

49. Lander, E. S. et al. Initial sequencing and analysis of the human genome. Nature 409 , of the mouse genome. Nature 420, 520-562 (2002). 860-921 (2001).

871 51. Lovell, P. V. et al. Conserved syntenic clusters of protein coding genes are missing in 872 birds. Genome Biol. 15, 565 (2014).

873 52. Xu, L. et al. OrthoVenn2: A web server for whole-genome comparison and annotation of 874 orthologous clusters across multiple species. Nucleic Acids Research vol. 47 W52-W58 875 (2019).

876 53. Hartley, G. \& O’Neill, R. Centromere repeats: Hidden gems of the genome. Genes vol. 10 
223 (2019).

878 54. Chueh, A. C., Wong, L. H., Wong, N. \& Choo, K. H. A. Variable and hierarchical size distribution of L1-retroelement-enriched CENP-A clusters within a functional human neocentromere. Hum. Mol. Genet. 14, 85-93 (2005).

55. Kuznetsova, I. S. et al. LINE-related component of mouse heterochromatin and complex chromocenters' composition. Chromosome Res. 24, 309-323 (2016).

56. Suh, A. The specific requirements for CR1 retrotransposition explain the scarcity of retrogenes in birds. J. Mol. Evol. 81, 18-20 (2015).

57. Benson, G. Tandem Repeats Finder: A program to analyze DNA sequences. Nucleic Acids Research vol. 27 573-580 (1999).

58. Igawa, T. et al. Inbreeding ratio and genetic relationships among strains of the Western

59. Ford, L. S. \& Cannatella, D. C. The major clades of frogs. Herpetological Monographs. 7, 94-117 (1993).

60. Bhutkar, A. et al. Chromosomal rearrangement inferred from comparisons of 12 Drosophila genomes. Genetics 179, 1657-1680 (2008).

61. Pyron, R. A. Divergence time estimation using fossils as terminal taxa and the origins of

64. Deakin, J. E. \& Ezaz, T. Understanding the evolution of reptile chromosomes through Lissamphibia. Syst. Biol. 60, 466-481 (2011).

62. Schubert, I. \& Lysak, M. A. Interpretation of karyotype evolution should consider applications of combined cytogenetics and genomics approaches. Cytogenet. Genome Res. 157, 7-20 (2019). 
65. Maruyama, T. \& Imai, H. T. Evolutionary rate of the mammalian karyotype. J. Theor. Biol. 90, 111-121 (1981).

66. Olmo, E. Rate of chromosome changes and speciation in reptiles. Genetica 125, 185-203 (2005).

907 67. Jagannathan, M., Cummings, R. \& Yamashita, Y. M. A conserved function for 908 pericentromeric satellite DNA. Elife 7, (2018).

909 68. Edwards, N. S. \& Murray, A. W. Identification of Xenopus CENP-A and an associated centromeric DNA repeat. Molecular Biology of the Cell vol. 16 1800-1810 (2005).

69. Smith, O. K. et al. Identification and characterization of centromeric sequences in Xenopus laevis. Cold Spring Harbor Laboratory 2020.06.23.167643 (2020) doi:10.1101/2020.06.23.167643.

914 70. Penke, T. J. R., McKay, D. J., Strahl, B. D., Matera, A. G. \& Duronio, R. J. Direct interrogation of the role of H3K9 in metazoan heterochromatin function. Genes Dev. 30, $1866-1880$ (2016).

71. Di Giacomo, M. et al. Multiple epigenetic mechanisms and the piRNA pathway enforce LINE1 silencing during adult spermatogenesis. Mol. Cell 50, 601-608 (2013).

72. Kong, A. et al. A high-resolution recombination map of the human genome. Nat. Genet. 31, 241-247 (2002).

73. Backstrom, N. et al. The recombination landscape of the zebra finch Taeniopygia guttata

74. Dréau, A., Venu, V., Avdievich, E., Gaspar, L. \& Jones, F. C. Genome-wide recombination map construction from single individuals using linked-read sequencing. Nat. Commun. 10, 4309 (2019).

75. Shanfelter, A. F., Archambeault, S. L. \& White, M. A. Divergent fine-scale recombination landscapes between a freshwater and marine population of threespine stickleback fish. Genome Biol. Evol. 11, 1573-1585 (2019). 
76. Singhal, S. et al. Stable recombination hotspots in birds. Science 350, 928-932 (2015).

930 77. Jensen-Seaman, M. I. et al. Comparative recombination rates in the rat, mouse, and 931 human genomes. Genome Res. 14, 528-538 (2004).

932 78. Baker, Z. et al. Repeated losses of PRDM9-directed recombination despite the 933 conservation of PRDM9 across vertebrates. Elife 6, (2017).

934 79. Kuhl, L.-M. \& Vader, G. Kinetochores, cohesin, and DNA breaks: Controlling meiotic 935 recombination within pericentromeres. Yeast 36, 121-127 (2019).

936 80. Termolino, P., Cremona, G., Consiglio, M. F. \& Conicella, C. Insights into epigenetic 937 landscape of recombination-free regions. Chromosoma vol. 125 301-308 (2016).

938 81. Groenen, M. A. M. et al. A high-density SNP-based linkage map of the chicken genome reveals sequence features correlated with recombination rate. Genome Res. 19, 510-519

82. Duret, L. \& Galtier, N. Biased gene conversion and the evolution of mammalian genomic landscapes. Annu. Rev. Genomics Hum. Genet. 10, 285-311 (2009).

83. Galtier, N., Piganeau, G., Mouchiroud, D. \& Duret, L. GC-content evolution in mammalian genomes: the biased gene conversion hypothesis. Genetics 159, 907-911 (2001).

84. Meunier, J. \& Duret, L. Recombination drives the evolution of GC-content in the human genome. Mol. Biol. Evol. 21, 984-990 (2004).

85. Lam, B. S. \& Carroll, D. Tandemly repeated DNA sequences from Xenopus laevis. I. Studies on sequence organization and variation in satellite 1 DNA (741 base-pair repeat). J. Mol. Biol. 165, 567-585 (1983).

86. Cohen, S., Menut, S. \& Méchali, M. Regulated formation of extrachromosomal circular

87. Ogiwara, I. V-SINEs: A new superfamily of vertebrate SINEs that are widespread in vertebrate genomes and retain a strongly conserved segment within each repetitive unit. 
Genome Research vol. 12 316-324 (2002).

88. Rao, S. S. P. et al. A 3D map of the human genome at kilobase resolution reveals principles of chromatin looping. Cell 159, 1665-1680 (2014).

89. Rabl, C. Über Zelltheilung. Morphologisches Jahrbuch 214-330 (1885).

90. Muller, H., Gil, J., Jr \& Drinnenberg, I. A. The impact of centromeres on spatial genome architecture. Trends Genet. 35, 565-578 (2019).

91. Therizols, P., Duong, T., Dujon, B., Zimmer, C. \& Fabre, E. Chromosome arm length and nuclear constraints determine the dynamic relationship of yeast subtelomeres. Proc. Natl.

92. Buttrick, G. J. et al. Nsk1 ensures accurate chromosome segregation by promoting association of kinetochores to spindle poles during anaphase B. Mol. Biol. Cell 22, 44864502 (2011).

93. Stevens, T. J. et al. 3D structures of individual mammalian genomes studied by single-cell Hi-C. Nature 544, 59-64 (2017).

94. Dudchenko, O. et al. De novo assembly of the Aedes aegypti genome using Hi-C yields chromosome-length scaffolds. Science 356, 92-95 (2017).

95. Duan, Z. et al. A three-dimensional model of the yeast genome. Nature vol. 465 363-367 (2010).

96. Armstrong, S. J., Franklin, F. C. \& Jones, G. H. Nucleolus-associated telomere clustering

97. Mascher, M. et al. A chromosome conformation capture ordered sequence of the barley and pairing precede meiotic chromosome synapsis in Arabidopsis thaliana. J. Cell Sci. genome. Nature 544, 427-433 (2017).

98. Cowan, C. R., Carlton, P. M. \& Cande, W. Z. The polar arrangement of telomeres in interphase and meiosis. Rabl organization and the bouquet. Plant Physiol. 125, 532-538 (2001). 
99. Varoquaux, N. et al. Accurate identification of centromere locations in yeast genomes using Hi-C. Nucleic Acids Res. 43, 5331-5339 (2015).

100. Rowley, M. J. \& Corces, V. G. Organizational principles of 3D genome architecture. Nat. Rev. Genet. 19, 789-800 (2018).

101. Lu, J. Y. et al. Homotypic clustering of $L 1$ and B1/Alu repeats compartmentalizes the 3D genome. Cell Res. (2021) doi:10.1038/s41422-020-00466-6.

102. Fishman, V. et al. 3D organization of chicken genome demonstrates evolutionary conservation of topologically associated domains and highlights unique architecture of erythrocytes' chromatin. Nucleic Acids Research vol. 47 648-665 (2019).

103. Kaaij, L. J. T., van der Weide, R. H., Ketting, R. F. \& de Wit, E. Systemic loss and gain of chromatin architecture throughout zebrafish development. Cell Rep. 24, 1-10.e4 (2018).

104. Eagen, K. P., Aiden, E. L. \& Kornberg, R. D. Polycomb-mediated chromatin loops revealed by a subkilobase-resolution chromatin interaction map. Proc. Natl. Acad. Sci. U. S. A. 114,

105. Dong, P. et al. 3D chromatin architecture of large plant genomes determined by local A/B compartments. Mol. Plant 10, 1497-1509 (2017).

106. Francke, U. 2012 William Allan Award: Adventures in cytogenetics. Am. J. Hum. Genet. 92, 325-337 (2013).

107. Uno, Y. et al. Diversity in the origins of sex chromosomes in anurans inferred from comparative mapping of sexual differentiation genes for three species of the Raninae and Xenopodinae. Chromosome Res. 16, 999-1011 (2008).

108. Uno, Y. et al. Inference of the protokaryotypes of amniotes and tetrapods and the

109. Parada, L. A., McQueen, P. G., Munson, P. J. \& Misteli, T. Conservation of relative evolutionary processes of microchromosomes from comparative gene mapping. PLoS One 7, e53027 (2012).

\footnotetext{
chromosome positioning in normal and cancer cells. Curr. Biol. 12, 1692-1697 (2002).
} 
110. Parada, L. A., McQueen, P. G. \& Misteli, T. Tissue-specific spatial organization of genomes. Genome Biol. 5, R44 (2004).

111. Lieberman-Aiden, E. et al. Comprehensive mapping of long-range interactions reveals folding principles of the human genome. Science 326, 289-293 (2009).

112. Uno, Y., Nishida, C., Takagi, C., Ueno, N. \& Matsuda, Y. Homoeologous chromosomes of Xenopus laevis are highly conserved after whole-genome duplication. Heredity vol. 111 430-436 (2013).

113. Rosin, L. F. et al. Chromosome territory formation attenuates the translocation potential of cells. Elife 8, (2019).

114. Bright, A. R. et al. Combinatorial transcription factor activities on open chromatin induce embryonic heterogeneity in vertebrates. EMBO J. 40, e104913 (2021).

115. Kakebeen, A. D., Chitsazan, A. D., Williams, M. C., Saunders, L. M. \& Wills, A. E. Chromatin accessibility dynamics and single cell RNA-Seq reveal new regulators of regeneration in neural progenitors. Elife 9, (2020).

116. Elinson, R. P. Metamorphosis in a frog that does not have a tadpole. Curr. Top. Dev. Biol. 103, 259-276 (2013).

117. del Pino, E. M. et al. A comparative analysis of frog early development. Proc. Natl. Acad. Sci. U. S. A. 104, 11882-11888 (2007).

118. Vargas, A. \& Del Pino, E. M. Analysis of cell size in the gastrula of ten frog species reveals a correlation of egg with cell sizes, and a conserved pattern of small cells in the marginal zone. J. Exp. Zool. B Mol. Dev. Evol. 328, 88-96 (2017).

119. Oswald, P. et al. Locality, time and heterozygosity affect chytrid infection in yellow-bellied toads. Dis. Aquat. Organ. 142, 225-237 (2020).

120. Alford, R. A., Dixon, P. M. \& Pechmann, J. H. Ecology. Global amphibian population declines. Nature vol. 412 499-500 (2001).

121. Leung, B. et al. Clustered versus catastrophic global vertebrate declines. Nature 588, 267- 
271 (2020).

1034

1035

1036

1037

1038

1039

1040

1041

1042

1043

1044

1045

1046

1047

1048

1049

1050

1051

1052

1053

1054

1055

1056

1057

1058

122. Weisenfeld, N. I., Kumar, V., Shah, P., Church, D. M. \& Jaffe, D. B. Direct determination of diploid genome sequences. Genome Res. 27, 757-767 (2017).

123. Ye, C., Hill, C. M., Wu, S., Ruan, J. \& Ma, Z. S. DBG2OLC: Efficient assembly of large genomes using long erroneous reads of the third generation sequencing technologies. Sci. Rep. 6, 31900 (2016).

124. Koren, S. et al. Canu: Scalable and accurate long-read assembly via adaptive k-mer weighting and repeat separation. Genome Res. 27, 722-736 (2017).

125. Kurtz, S. et al. Versatile and open software for comparing large genomes. Genome Biol. 5, R12 (2004).

126. Chakraborty, M., Baldwin-Brown, J. G., Long, A. D. \& Emerson, J. J. Contiguous and accurate de novo assembly of metazoan genomes with modest long read coverage. Nucleic Acids Research gkw654 (2016) doi:10.1093/nar/gkw654.

127. Boetzer, M., Henkel, C. V., Jansen, H. J., Butler, D. \& Pirovano, W. Scaffolding preassembled contigs using SSPACE. Bioinformatics 27, 578-579 (2011).

128. Durand, N. C. et al. Juicer provides a one-click system for analyzing loop-resolution Hi-C experiments. Cell Syst 3, 95-98 (2016).

129. Durand, N. C. et al. Juicebox provides a visualization system for Hi-C contact maps with unlimited zoom. Cell Systems vol. 3 99-101 (2016).

130. Dudchenko, O., Shamim, M. S., Batra, S. S. \& Durand, N. C. The Juicebox Assembly Tools module facilitates de novo assembly of mammalian genomes with chromosomelength scaffolds for under \$1000. Biorxiv (2018).

131. Chin, C.-S. et al. Nonhybrid, finished microbial genome assemblies from long-read SMRT sequencing data. Nat. Methods 10, 563-569 (2013).

132. Walker, B. J. et al. Pilon: An integrated tool for comprehensive microbial variant detection and genome assembly improvement. PLOS ONE vol. 9 e112963 (2014). 
1059

1060

1061

1062

1063

1064

1065

1066

1067

1068

1069

1070

1071

1072

1073

1074

1075

1076

1077

1078

1079

1080

1081

1082

1083

1084

133. Garrison, E. \& Marth, G. Haplotype-based variant detection from short-read sequencing. arXiv [q-bio. GN] (2012).

134. Shu, S., Rokhsar, D., Goodstein, D., Hayes, D. \& Mitros, T. JGI Plant Genomics Gene Annotation Pipeline. https://www.osti.gov/biblio/1241222 (2014).

135. Grabherr, M. G. et al. Full-length transcriptome assembly from RNA-Seq data without a reference genome. Nat. Biotechnol. 29, 644-652 (2011).

136. Haas, B. J. et al. De novo transcript sequence reconstruction from RNA-seq using the Trinity platform for reference generation and analysis. Nat. Protoc. 8, 1494-1512 (2013).

137. Smit, A. F. A. \& Hubley, R. RepeatModeler Open-1.0. (2008-2015).

138. Jurka, J. et al. Repbase Update, a database of eukaryotic repetitive elements. Cytogenet. Genome Res. 110, 462-467 (2005).

139. Smit, A. F. A., Hubley, R. \& Green, P. RepeatMasker Open-4.0. (2013-2015).

140. Chapman, J. A. et al. Meraculous: De novo genome assembly with short paired-end reads. PLoS One 6, e23501 (2011).

141. Goltsman, E., Ho, I. \& Rokhsar, D. Meraculous-2D: Haplotype-sensitive assembly of highly heterozygous genomes. arXiv [q-bio.GN] (2017).

142. Putnam, N. H. et al. Chromosome-scale shotgun assembly using an in vitro method for long-range linkage. Genome Res. 26, 342-350 (2016).

143. English, A. C. et al. Mind the gap: upgrading genomes with Pacific Biosciences RS longread sequencing technology. PLoS One 7, e47768 (2012).

144. Kajitani, R. et al. Efficient de novo assembly of highly heterozygous genomes from wholegenome shotgun short reads. Genome Res. 24, 1384-1395 (2014).

145. Mudd, A. B., Bredeson, J. V., Baum, R., Hockemeyer, D. \& Rokhsar, D. S. Analysis of muntjac deer genome and chromatin architecture reveals rapid karyotype evolution. Communications Biology vol. 3 (2020).

146. Paten, B. et al. Cactus: Algorithms for genome multiple sequence alignment. Genome Res. 
21, 1512-1528 (2011).

1086

1087

1088

1089

1090

1091

1092

1093

1094

1095

1096

1097

1098

1099

1100

1101

1102

1103

1104

1105

1106

1107

1108

1109

1110

147. Blanchette, M. et al. Aligning multiple genomic sequences with the threaded blockset aligner. Genome Res. 14, 708-715 (2004).

148. Kumar, S., Stecher, G., Suleski, M. \& Hedges, S. B. TimeTree: A resource for timelines, timetrees, and divergence times. Mol. Biol. Evol. 34, 1812-1819 (2017).

149. Kiełbasa, S. M., Wan, R., Sato, K., Horton, P. \& Frith, M. C. Adaptive seeds tame genomic sequence comparison. Genome Res. 21, 487-493 (2011).

150. Stamatakis, A. RAxML version 8: a tool for phylogenetic analysis and post-analysis of large phylogenies. Bioinformatics 30, 1312-1313 (2014).

151. Kumar, S., Stecher, G. \& Tamura, K. MEGA7: Molecular Evolutionary Genetics Analysis version 7.0 for bigger datasets. Mol. Biol. Evol. 33, 1870-1874 (2016).

152. Tamura, K. et al. Estimating divergence times in large molecular phylogenies. Proc. Natl. Acad. Sci. U. S. A. 109, 19333-19338 (2012).

153. Krzywinski, M. et al. Circos: an information aesthetic for comparative genomics. Genome Res. 19, 1639-1645 (2009).

154. Dondoshansky, I. \& Wolf, Y. Blastclust (NCBI Software Development Toolkit). ScienceOpen https://www.scienceopen.com/document?vid=b654ab9a-231d-410a-832d37c7c7bc7165 (2002).

155. Camacho, C. et al. BLAST+: Architecture and applications. BMC Bioinformatics 10, 421 (2009).

156. Li, H. Aligning sequence reads, clone sequences and assembly contigs with BWA-MEM. arXiv [q-bio. GN] (2013).

157. Li, H. et al. The Sequence Alignment/Map format and SAMtools. Bioinformatics 25, 20782079 (2009).

158. Quinlan, A. R. BEDTools: The Swiss-army tool for genome feature analysis. Curr. Protoc. Bioinformatics 47, 11.12.1-34 (2014). 
159. Bredeson, J. V. et al. Sequencing wild and cultivated cassava and related species reveals extensive interspecific hybridization and genetic diversity. Nat. Biotechnol. 34, 562-570 (2016).

160. Bolger, A. M., Lohse, M. \& Usadel, B. Trimmomatic: a flexible trimmer for Illumina sequence data. Bioinformatics vol. 30 2114-2120 (2014).

161. Li, H. Minimap2: Pairwise alignment for nucleotide sequences. Bioinformatics 34, 30943100 (2018).

162. Ramírez, F. et al. deepTools2: a next generation web server for deep-sequencing data analysis. Nucleic Acids Res. 44, W160-5 (2016).

163. Van Ooijen, J. W. Multipoint maximum likelihood mapping in a full-sib family of an outbreeding species. Genet. Res. 93, 343-349 (2011).

164. Myers, S., Bottolo, L., Freeman, C., McVean, G. \& Donnelly, P. A fine-scale map of recombination rates and hotspots across the human genome. Science $310,321-324$ (2005).

165. Shifman, S. et al. A high-resolution single nucleotide polymorphism genetic map of the mouse genome. PLoS Biol. 4, e395 (2006).

166. Knight, P. A. \& Ruiz, D. A fast algorithm for matrix balancing. IMA J. Numer. Anal. 33, 1029-1047 (2012).

167. R Core Team. R Core Team. R: A language and environment for statistical computing. Foundation for Statistical Computing (2013).

168. Tang, H. et al. Synteny and collinearity in plant genomes. Science 320, 486-488 (2008). 
1133 We thank Karen Lundy and the Functional Genomics Laboratory at the University of California

1134 Berkeley for running quality control on extracted DNA and RNA and for preparing Illumina short-

1135 insert libraries; Oanh Nguyen and the DNA Technologies and Expression Analysis Cores at the

1136 University of California Davis Genome Center for preparing and sequencing PacBio libraries;

1137 Dovetail Genomics for providing the HiC library preparation kit, running quality control on $\mathrm{HiC}$

1138 libraries, and preparing and sequencing HiC libraries; Shana McDevitt and the Vincent J.

1139 Coates Genomics Sequencing Laboratory at the University of California Berkeley for

1140 sequencing $\mathrm{HiC}$ and Illumina short-insert libraries; Shengqiang Shu for advice on the use of the

1141 IGC annotation pipeline. We thank Rick Elinson for providing E. coqui frogs and tissues. We

1142 thank Gary Gorbsky from the Oklahoma Medical Research Foundation and Marko Horb and the

1143 National Xenopus Resource at the MBL for providing the XTN-6 cell lines. Finally, we thank

1144 Chunhui Hou and colleagues for permission to access their HiC data prior to publication.

1147 This study was supported by NIH grants R01HD080708 to D.S.R.; R01GM086321,

1148 R01HD065705 to D.S.R. and R.M.H.; R35GM127069 to R.M.H.; R35 GM118183 to R.B.H.

1149 A.B.M. was supported by NIH grants T32GM007127 and T32HG000047 and a David L. Boren

1150 Fellowship. D.S.R. is grateful for support from the Marthella Foskett Brown Chair in Biological

1151 Sciences; R.M.H., the C.H. Li Distinguished Chair in Molecular and Cell Biology; and R.H., the

1152 Flora Lamson Hewlett chair in biochemistry. A.F.S. and O.K.S. were supported by

1153 R01GM074728, O.K.S. by NIH T32 GM113854-02 and NSF GRFP; M.K.K. and M.L. by

1154 R01HD102186; J.H. by NSF grants DEB-1701591 and DBI-1702263; M.L., a Women in Science

1155 Fellowship; T.K. by the Basic Science Research Program, National Research Foundation of 
Korea (NRF), Ministry of Education (2018R1A6A1A03025810), Future-leading Project Research

1157 Fund (1.200094.01) of UNIST and the Institute for Basic Science (IBS-R022-D1); J.B.W. and

1158 H.S.P. by R01GM104853, R01HD085901; M.J.R. by NSF IOS-0910112; Smithsonian Tropical

1159 Research Institute; Clark Hubbs Regents Professorship; L.M.S. by the "Centre National de la

1160 Recherche Scientifique" (PEPS ExoMod "Triton") and the "Muséum National d'Histoire

1161 Naturelle" (Action Transversale du Muséum “Cycles biologiques: Evolution et adaptation”) and a

1162 Scientific council post-doctoral position to G.K.

1164 This work used the Vincent J. Coates Genomics Sequencing Laboratory at the University of 1165 California Berkeley, supported by NIH grant S10OD018174, and the DNA Technologies and 1166 Expression Analysis Cores at the University of California Davis Genome Center, supported by

1167 NIH grant S10OD010786. This research used the National Energy Research Scientific

1168 Computing Center, a Department of Energy Office of Science User Facility supported by 1169 contract number DE-AC02-05CH11231. LMS acknowledges the "Ecole Normale Supérieure de

1170 PARIS" genomic platform for RNA-sequencing and the PCIA high performance computing 1171 platform at "Muséum National d'Histoire Naturelle".

1175 J.V.B., A.B.M., S.M-R., T.M., R.M.H., and D.S.R. wrote the manuscript with feedback from M.L., 1176 H.P.S., J.H., J.B.L., J.B.W., M.J.R., O.K.S., D.R.B., M.G-P., J.H., N.B., T.K., L.M.S., R.H., J.S., 1177 M.K.K., A.F.S., and D.H. Genomes were assembled by J.V.B., S.S.B. (Xtr); A.B.M., and K.C.B. 1178 (other frogs). S.M-R., A.B.M., and G.K. assembled transcripts and annotated genomes. S.M-R. 1179 and J.V.B. assessed gene completeness; S.M-R. analyzed repeat and recombination 1180 landscapes. S.M-R. and J.P. identified centromeric repeats. O.K.S., G.A-F. and A.F.S. 
conducted ChIP-seq experiments and S.M-R. performed analysis. J.V.B. analyzed HiC features.

1182 T.M. constructed the linkage map. T.M. and J.V.B. analyzed heterozygosity. A.B.M. performed

1183 genome-wide comparisons. K.E.M. and R.H. examined Hbo metaphase spreads. M.K.K. and

1184 M.L. inbred Xtr frogs. R.M.H. (Xtr); M.G-P. (Epu); K.E.M. and R.H. (Hbo); M.L. and J.H. (Eco)

1185 collected frogs. R.M.H. (Xtr); M.G-P., H.S-P. (Epu); and D.R.B. (Eco) collected tissue samples.

1186 A.B.M., D.R.B. (Eco); J.B.L., and I.P. (Xtr) extracted DNA. A.B.M., S.M-R. (Epu); K.E.M., R.H.

1187 (Hbo); and L.M.S. (Eco) extracted RNA and libraries were prepared by A.B.M. (Epu). M.L., J.H.

1188 (Eco); K.E.M., and R.H. (Hbo) provided RNA-seq data. T.K., M.J.R., J.B.W. (Epu); and J.B.L.

1189 (Xtr) coordinated sequencing. C.P., J.G., and J.S. prepared and sequenced 10x Genomics,

1190 PacBio, and Illumina mate-pair libraries. D.H. prepared HiC libraries. R.D.D. and J.H.M.

1191 provided early access to the Pad assembly. N.B. (Eco) provided bioinformatic support. L.M.S.

1192 led the Eco efforts. R.M.H. and D.S.R. led the project.

\section{Ethics Declarations}

1195 Competing Interests

1196 D.S.R. is a member of the Scientific Advisory Board of, and a minor shareholder in, Dovetail

1197 Genomics LLC, which provides as a service the high-throughput chromatin conformation

1198 capture (HiC) technology used in this study.

1199

1200 M.K.K. is President and co-founder of Victory Genomics, Inc. 

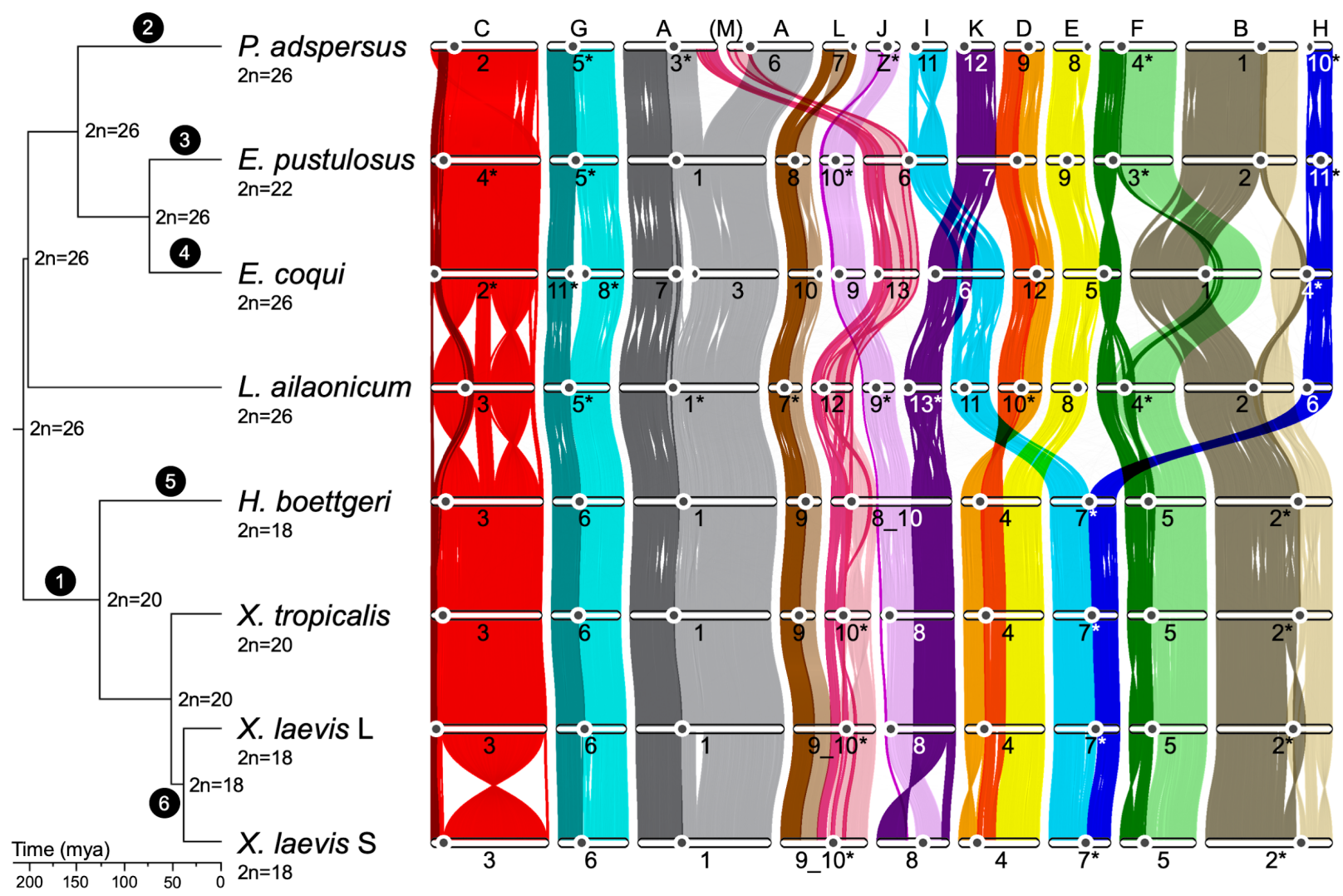

\section{Fig. 1 Phylogenetic tree and gene ortholog alignment.}

1203 The phylogenetic tree of the seven analyzed species, calculated from fourfold degenerate sites and 1204 divergence time confidence intervals, was visualized with FigTree (commit 901211e; https://github.com/rambaut/figtree). The ancestral karyotype at each node was labeled on the tree. The alignment plot was generated with jcvi.graphics.karyotype ${ }^{168}$ (v0.8.12; https://github.com/tanghaibao/jcvi) using the 7,292 described chromosome one-to-one gene orthologs from OrthoVenn2 $\left(\right.$ ref. $\left.^{52}\right)$, followed by visual filtering of single stray orthologs. The pericentromeric region based on $\mathrm{HiC}$ inference was represented with a black circle on each chromosome. The ancestral chromosomes ( $A$ to $M)$ were labeled at the top of the alignment based on the corresponding region in $P$. adspersus. The alignments for each ancestral chromosome were colored uniquely, with those upstream and downstream of the $X$. tropicalis centromeric satellite repeat from tandem repeat analysis shaded with a light versus dark shade of the ancestral chromosome color. Chromosomes labeled with an asterisk were reverse complemented in this

1214 image relative to the orientation in the assembly. Black circles with white text reference chromosome 1215 changes outlined in Table 1. 

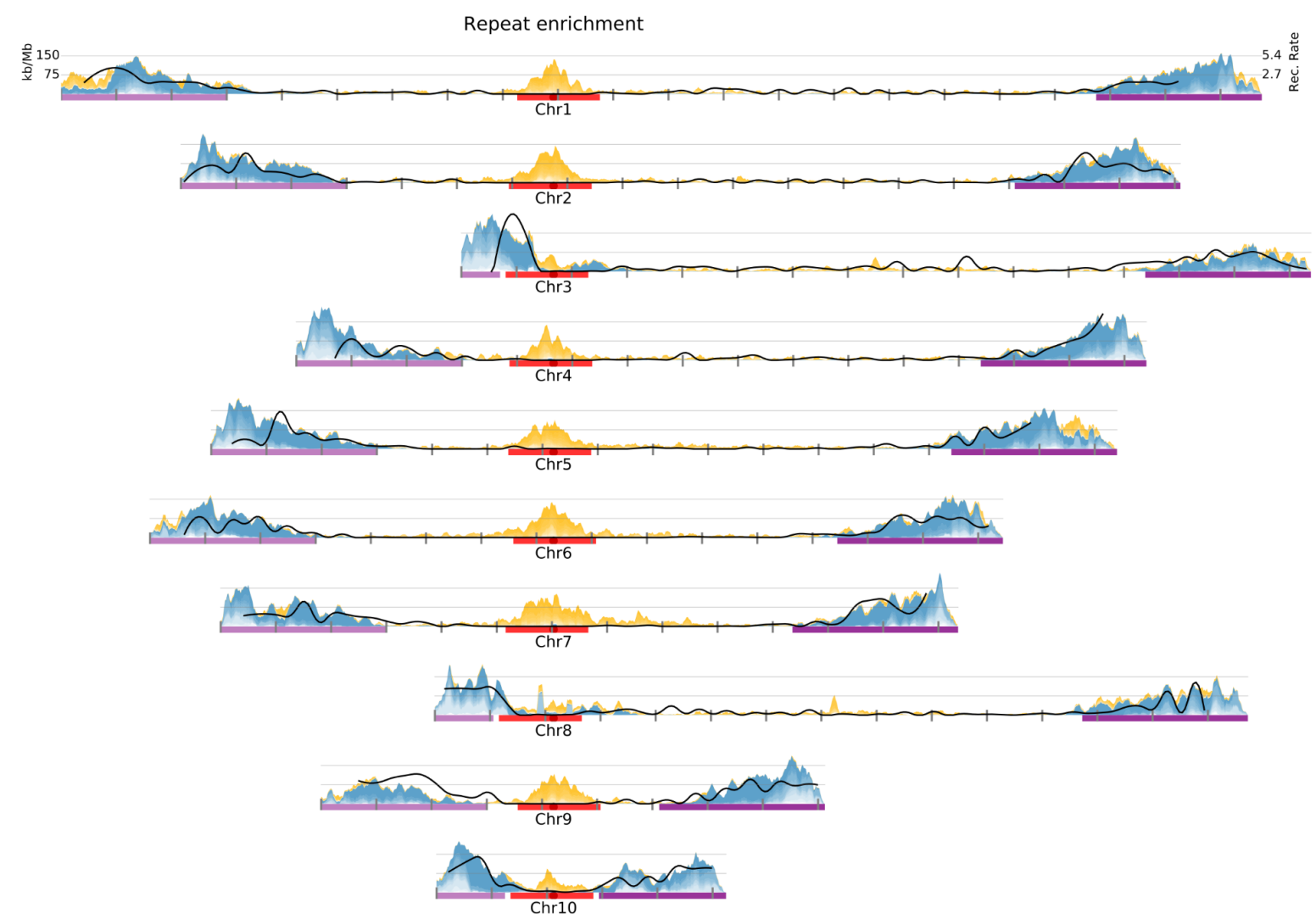

1217 Fig. 2 Density of pericentromeric and subtelomeric repeats in Xenopus tropicalis.

1218 Pericentromeric boundaries (red) and subtelomeric boundaries (purple) were used to obtain enriched

1219 repeats excluding chromosomes with short p-arms (chromosomes 3, 8, and 10). Pericentromeric repeats

1220 (yellow) correspond to selected subsets of non-LTR retrotransposons (CR1, L1, and Penelope), LTR

1221 retrotransposons (Ty3), and DNA transposons (PiggyBac and Harbinger). Subtelomeric enriched repeats

1222 (blue) correspond mainly to Satellite repeats and LTR retrotransposons (Ty3, Ngaro). Chromosomes are

1223 centered by the position of centromeric tandem repeats (black dot and dotted vertical line). The rate of

1224 recombination $(\mathrm{cM} / \mathrm{Mb})$ is shown as a solid black line. Tick marks indicate $10 \mathrm{Mb}$ blocks (Supplementary

1225 Fig. 16). 
A

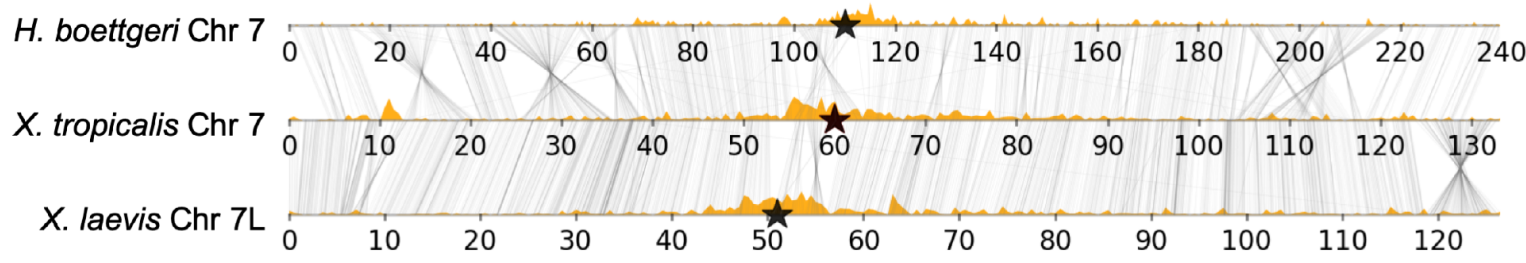

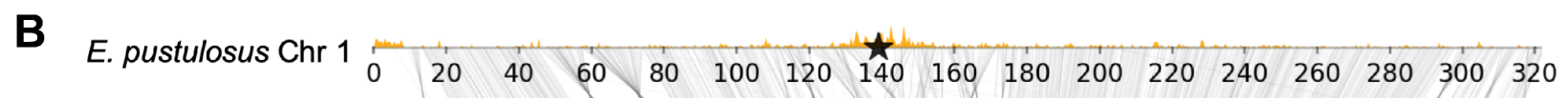

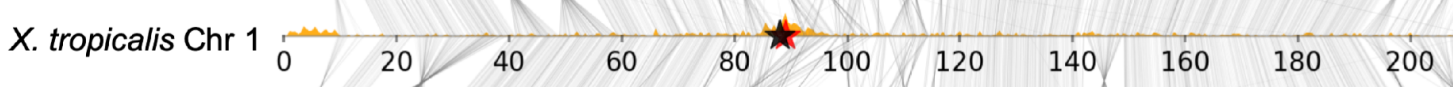

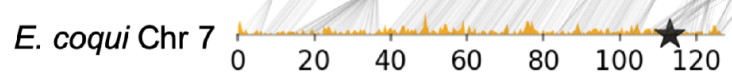

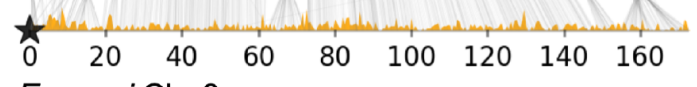

E. coqui $\mathrm{Chr} 3$

C

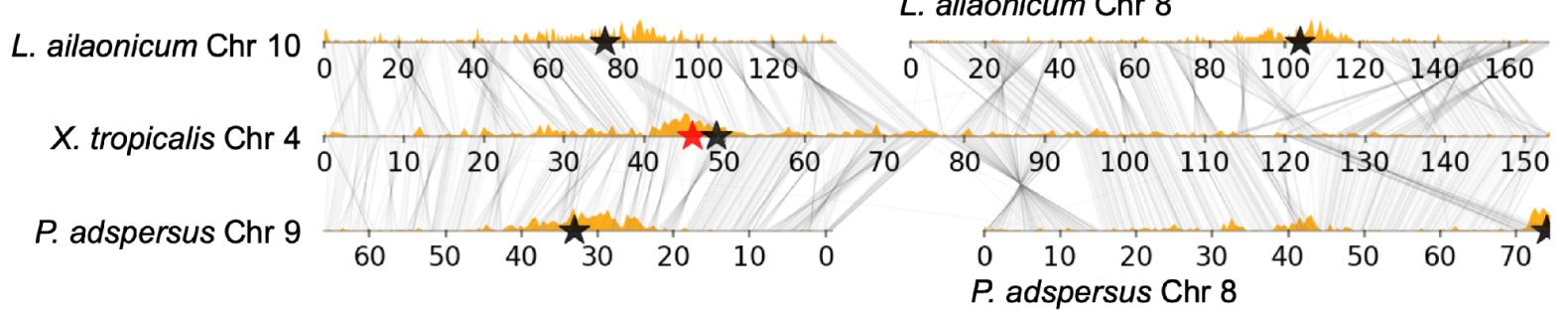

1227 Fig. 3 Subtelomeric repeats highlight regions of chromosome fusion.

1228 Examples of $(\mathbf{A})$ conserved chromosome structure and pericentromere maintenance, $(\mathbf{B})$ a Robertsonian

1229 translocation in the lineage leading to $E$. coqui, and (C) an end-to-end fusion that occurred in the lineage

1230 giving rise to $X$. tropicalis and subsequent pericentromere loss. The analyzed species were visualized

1231 with a custom script, alignment_plots.py (v1.0; https://github.com/abmudd/Assembly). For each plot, the

$1232 \mathrm{HiC}$ inference-based pericentromeric regions are depicted with black stars, the $X$. tropicalis centromeric

1233 satellite repeat from tandem repeat analysis with a red star, the density of L1 repeats per chromosome

1234 with light brown histograms, and the runs of collinearity containing at least one kb of aligned sequence

1235 between the species with connecting black lines. 
A
Do

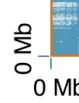

$\sum_{\substack{0 \\ \delta^{-}}}^{0}$
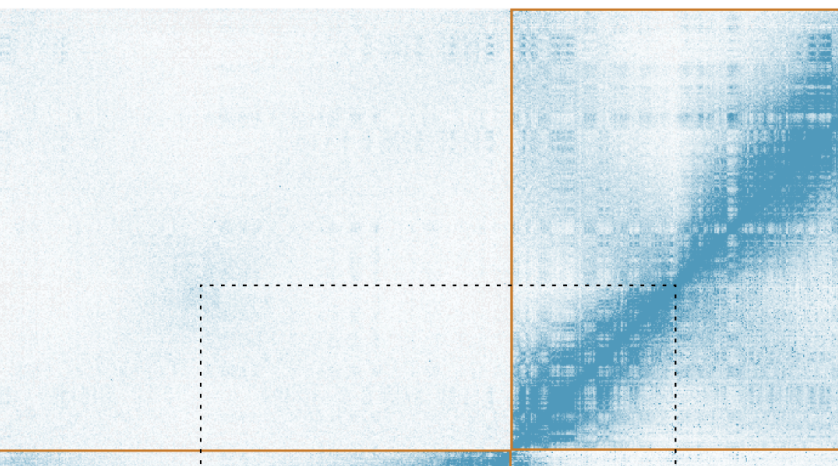

B

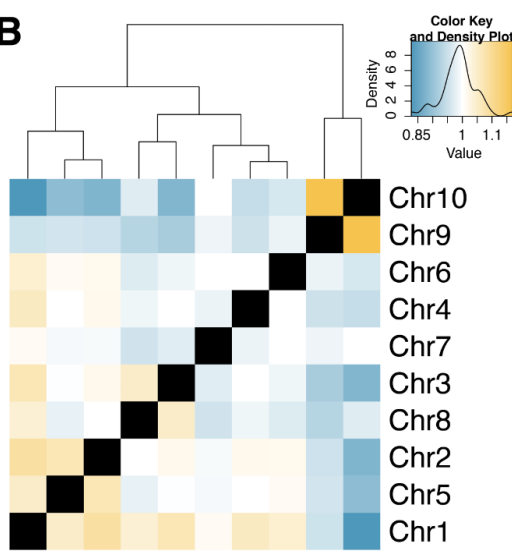

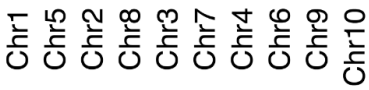

C

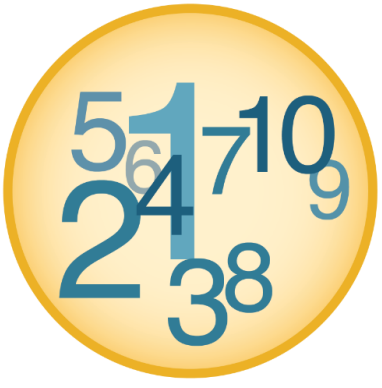

Fig. 4 Organization of $X$. tropicalis chromosomes into Rabl configuration and distinct nuclear territories.

(A) $\mathrm{HiC}$ contact matrices at $500 \mathrm{~kb}$ resolution for chromosomes 1 and 2 (lower-left and upper-right gold boxes, respectively) showing features of the three-dimensional chromatin architecture within $X$. tropicalis blood cell nuclei. Blue pixels represent chromatin contacts between $X-Y$ pairs of genomic loci, and their intensity is proportional to their contact frequency. $\mathrm{HiC}$ read pairs are mapped stringently (MapQ $\geq 30$ ) above the diagonal and permissively $(\mathrm{MapQ} \geq 0)$ below the diagonal. The characteristic $A / B$ compartment ("checkerboard") and Rabl-like inter-arm ("angel wing") contact patterns within each chromosome are evident. Above the diagonal, an increased frequency of inter-chromosomal chromatin contacts is observed between pericentromeres (connected by dotted lines) and between chromosome arms, suggesting a centromere-clustered organization of chromosomes in Rabl configuration. Below the diagonal, high-intensity pixels not present above the diagonal are present near the ends of chromosomes, suggesting a telomere-proximal spatial bias in the distributions of similar genomic repeats. See

Supplementary Fig. 1D for a plot showing all chromosomes. (B) Chromosome territories within the 
1251 nucleus. Yellow, white, and blue colors indicate the normalized relative enrichment, parity, and depletion

1252 of chromatin contacts between non-homologous chromosomes in the nucleus. For example,

1253 Chromosome 1 exhibits higher relative contact frequencies with all chromosomes except chromosomes

1254 Chr7, Chr9, and Chr10, which are generally depleted of contacts except among themselves. (MapQ $\geq 30$;

$1255 \chi^{2}(81, n=24,987,749)=3,049,787 ; p<2.2 \times 10^{-308}$; Relative range: $\left.0.82774-1.16834\right)$. (C) Schematic

1256 representation of chromosome territories within the nucleus. Chromosome number size is proportional to

1257 the number of enriched interactions. Darker and lighter colors indicate chromosomes nearer or distant to

1258 the reader, respectively. 
A

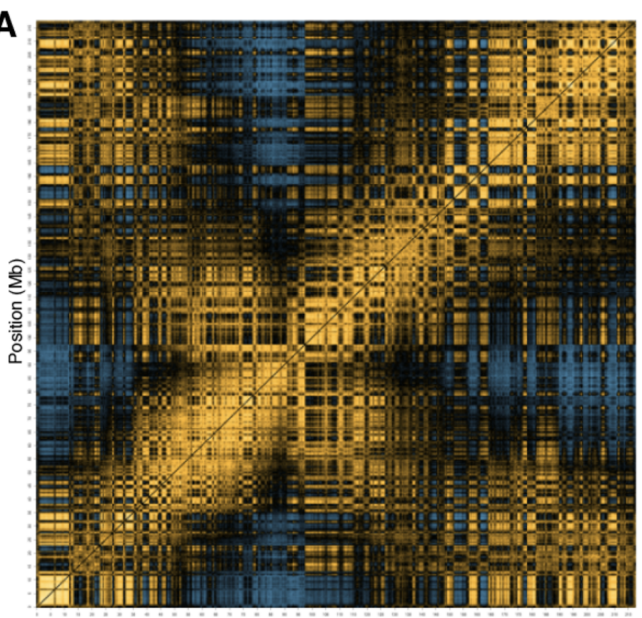

B

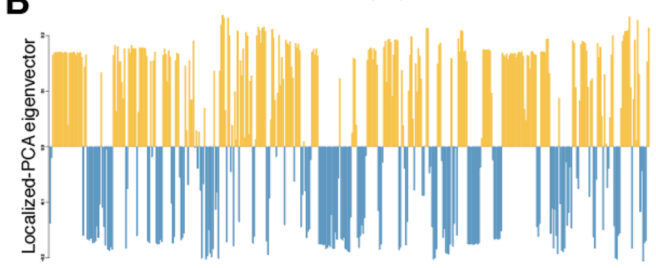

C
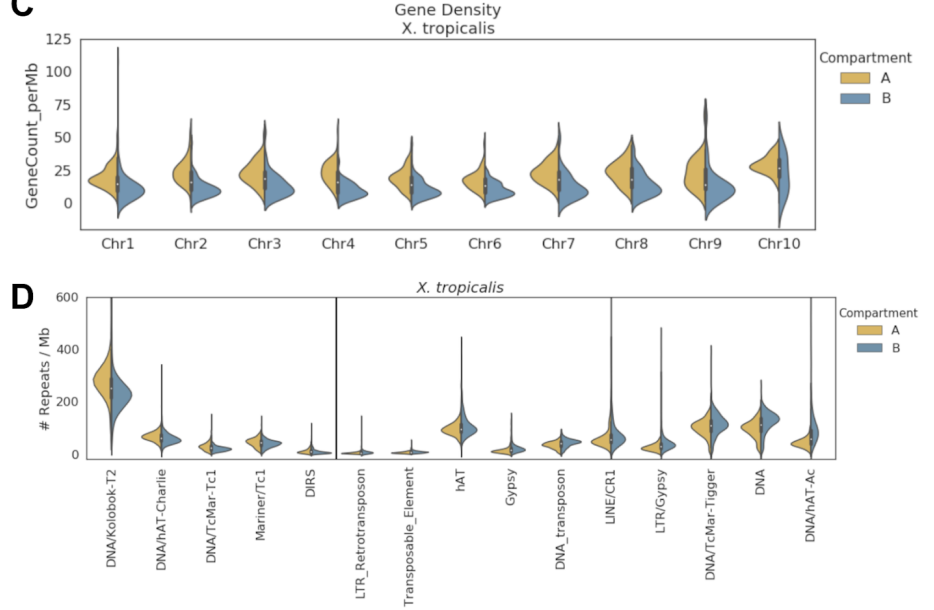

E

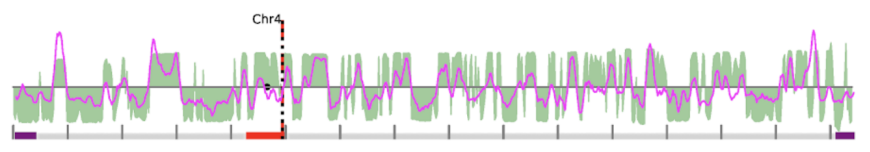

1260 Fig. 5 A/B compartment structure and gene/repeat densities.

1261 (A) Correlation matrix of intra-chromosomal HiC contact densities between all pairs of non-overlapping

$250 \mathrm{~kb}$ loci on chromosome 1. Yellow and blue pixels indicate correlation and anti-correlation,

respectively, and reveal which genomic loci occupy the same or different chromatin compartment. Black

1264 pixels indicate weak/no correlation. (B) The first principal component eigenvectors revealing compartment structure along chromosome 1, obtained by singular value decomposition of the correlation matrix in $\mathbf{A}$.

1266 Yellow (positive) and blue (negative) eigenvectors indicate regions of chromosome 1 partitioned into the

1267 A and B compartments, respectively. (C) Gene density distributions in A vs. B compartments per

1268 chromosome. (D) Repeat classes significantly enriched in A vs. B compartments. (E) The eigenvectors

1269 obtained from the $\mathrm{HiC}$ correlation matrix (PC1, green) and the eigenvectors from the repeat density matrix

1270 (PC3, pink) coincide at the transitions of predicted A/B compartments (Supplementary Fig. 16B). 


\section{Table 1 Organization and conservation of the 13 ancestral chromosomes of pipanuran genomes}

Phylogenetic position

(1) Stem pipid lineage

(2) $P$. adspersus lineage after divergence from $R$. temporaria

(3) E. pustulosus lineage after divergence from $E$. coqui

(4) E. coqui lineage after divergence from E. pustulosus

(5) $H$. boettgeri lineage after divergence from Xenopus

(6) X. laevis progenitor lineage after divergence from $X$. tropicalis

\section{Structural event}

$$
\begin{aligned}
& \mathrm{J}+\mathrm{K} \Rightarrow \mathrm{JK} \\
& \mathrm{D} .+\mathrm{E} . \Rightarrow \mathrm{D} . \mathrm{E} \\
& \mathrm{I}+\cdot \mathrm{H} \Rightarrow \mathrm{I} \cdot \mathrm{H} \text { (Rob. fusion) }
\end{aligned}
$$

$$
A+M \Rightarrow A 1 \cdot m 1+m 2 . A 2
$$

$\mathrm{M}+\mathrm{I} \Rightarrow \mathrm{M} . \mathrm{I}$ (Rob)

$\mathrm{K}+\mathrm{D} \Rightarrow \mathrm{K} . \mathrm{D}$ (Possible end-end)

$$
\begin{aligned}
& \mathrm{G} 1 \cdot \mathrm{G} 2 \Rightarrow \mathrm{G} 1 \cdot+\cdot \mathrm{G} 2 \text { (Rob. fission) } \\
& \mathrm{A} 1 \cdot \mathrm{A} 2 \Rightarrow \mathrm{A} 1 \cdot+\cdot \mathrm{A} 2 \text { (Rob. fission) } \\
& \mathrm{I}+\mathrm{K} \Rightarrow \mathrm{I} \cdot \mathrm{K}(\text { Rob. fusion }+ \text { inversion) } \\
& \mathrm{E}+\mathrm{F} 1 \cdot \mathrm{F} 2+\mathrm{B} 1 \cdot \mathrm{B} 2+\mathrm{H} \Rightarrow \mathrm{E} \cdot \mathrm{F} 1+\mathrm{F} 2 \cdot \mathrm{B} 2+\mathrm{B} 1 \cdot \mathrm{H}
\end{aligned}
$$

$\mathrm{M}+\mathrm{J} \bullet \mathrm{K} \Rightarrow \mathrm{MJK}$

$L+M \Rightarrow L M$

Rob, Robertsonian. Middle-dots (i.e., “•”) represent centromeres. Periods (i.e., “.”) represent translocation breakpoints. 


\section{Supplementary Information}

1272 (Provided in a separate document)

1273 Supplementary Fig. 1 Genome assembly and recovery of missing genes.

1274 Supplementary Fig. 2 Xenopus tropicalis genome-wide HiC contact map.

1275 Supplementary Fig. 3 GC landscape and tandem repeats.

1276 Supplementary Fig. 4 Comparison of gene content in assemblies of model vertebrates.

1277 Supplementary Fig. 5 PCA eigenvectors projected on genomic coordinates.

1278 Supplementary Fig. 6 Xenopus tropicalis Nigerian strain residual heterozygosity.

1279 Supplementary Fig. 7 Assembly and annotation of other frog species.

1280 Supplementary Fig. 8 Pairwise gene colinearity of frog genomes.

1281 Supplementary Fig. 9 Chromosome fusions in Xenopus laevis and Hymenochirus boettgeri.

1282 Supplementary Fig. 10 Estimating the positions of Xenopus tropicalis centromeres.

1283 Supplementary Fig. 11 Xenopus tropicalis recombination landscape.

1284 Supplementary Fig. 12 Distribution of Satellite repeats in Xenopus tropicalis.

1285 Supplementary Fig. 13 Correlates of recombination rate.

1286 Supplementary Fig. 14 Zebra finch subtelomeric tandem repeats.

1287 Supplementary Fig. 15 Microsatellite origin SINE/tRNA evolved into a microsatellite sequence.

1288 Supplementary Fig. 16 Xenopus tropicalis 3D chromatin structure and nuclear organization.

1289 Supplementary Table 1 Sequence Completeness.

1290 Supplementary Table 2 Transcript coverage of $X$. tropicalis assemblies $\mathrm{v} 9$ and $\mathrm{v} 10$.

1291 Supplementary Table 3 Xenopus tropicalis protein coding loci annotation summary statistics.

1292 Supplementary Table 4 Xenopus tropicalis repeat abundances.

1293 Supplementary Table 5 Summary of other frog genome assemblies.

1294 Supplementary Table 6 Summary of annotations for other frog genomes.

1295 Supplementary Table 7 BUSCO genome scores of other frog genome assemblies. 
1296 Supplementary Table 8 Ancestral chromosome fusions.

1297 Supplementary Table 9 N50 lengths for collinear runs of orthologous genes between frogs.

1298 Supplementary Table 10 Four-fold degeneracy nucleotide divergence.

1299 Supplementary Table 11 Estimation of divergence times.

1300 Supplementary Table 12 Centromeric Associated Tandem Repeat monomer lengths and

1301 counts.

1302 Supplementary Table 13 Mapping statistics for ChIP-seq samples.

1303 Supplementary Table 14 Correlates of recombination rate.

1304 Supplementary Table 15 Subtelomeric enrichment for tandem repeats.

1305 Supplementary Table 16 Correspondence of monomer sequence with annotated repeat 1306 elements.

1307 Supplementary Table 17 Copy counts of 52-mer minisatellite.

1308 Supplementary Table 18 Quantification of Rabl structure strength and significance.

1309 Supplementary Table 19 Contact enrichment between chromosomes and chromosome arms.

1310 Supplementary Table 20 Relative enrichment of HiC contacts between chromosomes.

1311 Supplementary Note 1 High-throughput sequencing, Xenopus tropicalis.

1312 Supplementary Note 2 Xenopus tropicalis genome assembly and annotation.

1313 Supplementary Note 3 Additional chromosome-scale frog assemblies.

1314 Supplementary Note 4 Comparative analysis.

1315 Supplementary Note 5 Genome analysis. 


\section{Supplementary Data Files}

\section{Supplementary Data 1: Table of sequencing data.}

1318 An MS Excel file summarizing the sequencing data used to construct the six frog genome

1319 assemblies new or updated in this study, as well as the RNA-seq data used for annotating their

1320 protein-coding genes. The $X$. tropicalis ChIP-seq data are also included.

1322 Supplementary Data 2: Genetic markers.

1323 An MS Excel file containing the marker number, locus identifier, chromosome name,

1324 centiMorgan position, and chromosome coordinate for each genetic marker in the $F_{2} X$.

1325 tropicalis genetic linkage map. 